LTH 952

July 2012

LPN12-083

\title{
Resummed small-x and first-moment evolution of fragmentation functions in perturbative QCD
}

\author{
C.-H. Kom ${ }^{a}$, A. $\operatorname{Vogt}^{a}$ and K. Yeats ${ }^{b}$ \\ ${ }^{a}$ Department of Mathematical Sciences, University of Liverpool \\ Liverpool L69 3BX, United Kingdom \\ ${ }^{b}$ Department of Mathematics, Simon Fraser University \\ University Drive, Burnaby, BC, V5A 1S6, Canada
}

\begin{abstract}
We study the splitting functions for the evolution of fragmentation distributions and the coefficient functions for single-hadron production in semi-inclusive $e^{+} e^{-}$annihilation in massless perturbative QCD for small values of the momentum fraction and scaling variable $x$, where their fixed-order approximations are completely destabilized by huge double logarithms of the form $\alpha_{\mathrm{s}}^{n} x^{-1} \ln ^{2 n-a} x$. Complete analytic all-order expressions in Mellin- $N$ space are presented for the resummation of these terms at the next-to-next-to-leading logarithmic accuracy. The poles for the first moments, related to the evolution of hadron multiplicities, and the small- $x$ instabilities of the next-to-leading order splitting and coefficient functions are removed by this resummation, which leads to an oscillatory small $x$ behaviour and functions that can be used at $N=1$ and down to extremely small values of $x$. First steps are presented towards extending these results to the higher accuracy required for an all- $x$ combination with the state-of-the-art next-to-next-to-leading order large- $x$ results.
\end{abstract}




\section{Introduction}

The fragmentation distributions, or parton fragmentation functions, $D_{p}^{h}\left(x, \mu^{2}\right)$ encode the probability of a final-state parton $p$ in a hard scattering process to end up in (or fragment into) a hadron $h$ which carries a fraction $x$ of the momentum of the parent (anti-) quark $q$ or gluon $g$. Like their even more important initial-state counterparts, the parton distributions of hadrons $f_{p}^{h}\left(x, \mu^{2}\right)$, these quantities include long-distance information and are thus not calculable in perturbative Quantum Chromodynamics (QCD). Their dependence on the fragmentation (final-state mass factorization) scale $\mu$, to be chosen of the order of a physical hard scale in the scattering process under consideration, is however calculable via the renormalization-group evolution equations

$$
\frac{\partial}{\partial \ln \mu^{2}} D_{i}^{h}\left(x, \mu^{2}\right)=\sum_{j=\mathrm{q}, \mathrm{g}}\left[P_{j i}^{T}\left(\alpha_{\mathrm{s}}\left(\mu^{2}\right)\right) \otimes D_{j}^{h}\left(\mu^{2}\right)\right](x) .
$$

Here $\otimes$ denotes the standard Mellin convolution in the first arguments,

$$
\left[f_{1}\left(\mu^{2}\right) \otimes f_{2}\left(\mu^{2}\right)\right](x) \equiv \int_{x}^{1} \frac{d y}{y} f_{1}\left(y, \mu^{2}\right) f_{2}\left(\frac{x}{y}, \mu^{2}\right),
$$

which is reduced to a simple product by the transformation to Mellin moments,

$$
f_{i}\left(N, \mu^{2}\right)=\int_{0}^{1} d x x^{N-1} f_{i}\left(x, \mu^{2}\right) .
$$

The final-state ('timelike') splitting functions $P_{j i}^{T}$ in Eq. (1.1) admit an expansion in power of the renormalized strong coupling constant, here normalized as $a_{\mathrm{s}} \equiv \alpha_{\mathrm{s}}\left(\mu^{2}\right) /(4 \pi)$,

$$
P_{j i}^{T}\left(x, \alpha_{\mathrm{s}}\left(\mu^{2}\right)\right)=a_{\mathrm{s}} P_{j i}^{T(0)}(x)+a_{\mathrm{s}}^{2} P_{j i}^{T(1)}(x)+a_{\mathrm{s}}^{3} P_{j i}^{T(2)}(x)+\ldots,
$$

where we have, without loss of information, identified the coupling-constant renormalization and mass-factorization scales.

A benchmark process for parton fragmentation is semi-inclusive $e^{+} e^{-}$annihilation (SIA),

$$
e^{+} e^{-} \rightarrow \gamma^{*}, Z, H \rightarrow h+X
$$

which is closely related to deep-inelastic scattering (DIS), $e h \rightarrow e+X$, via the exchange of a virtual photon $\gamma^{*}$, Z-boson or Higgs particle. In the former (latter) case the four-momentum $q$ of the exchanged boson is timelike, $q^{2}>0$ (spacelike, $q^{2}<0$ ). $X$ stands for all hadronic final states allowed by quantum number conservation. The cross section for vector-boson exchange can be written as [1]

$$
\frac{1}{\sigma_{0}} \frac{d^{2} \sigma}{d x d \cos \theta}=\frac{3}{8}\left(1+\cos ^{2} \theta\right) F_{T}^{h}\left(x, Q^{2}\right)+\frac{3}{4} \sin ^{2} \theta F_{L}^{h}\left(x, Q^{2}\right)+\frac{3}{4} \cos \theta F_{A}^{h}\left(x, Q^{2}\right),
$$

where $\theta$ is the angle, in the center-of-mass (CM) frame, between the incoming electron beam and the hadron $h$ observed with four-momentum $p$, and the scaling variable is given by $x=2 p q / Q^{2}$ where $Q^{2} \equiv q^{2}$ in SIA. The dimensionless transverse $(T)$ and longitudinal $(L)$ fragmentation functions in Eq. (1.6), and the total fragmentation function $F_{I}^{h}=F_{T}^{h}+F_{L}^{h}$ obtained by integrating 
Eq. (1.6) over $\theta$, have been measured at LEP and earlier $e^{+} e^{-}$colliders, see Ref. [2] for a general overview. The interference of vector and axial-vector contributions leads to the parity-violating $\cos \theta$ term. The corresponding fragmentation function $F_{A}$ does not receive $1 / x$ contributions and will not be considered in this article; it should be ignored when Eq. (1.6) is referred to below.

Up to corrections suppressed by powers of the CM energy $\sqrt{s}=Q$, the fragmentation functions can be expressed in terms of the fragmentation distributions and coefficient functions $C_{a, i}$,

$$
F_{a}^{h}\left(x, Q^{2}\right)=\sum_{i=\mathrm{q}, \mathrm{g}}\left[C_{a, i}\left(Q^{2}\right) \otimes D_{i}^{h}\left(Q^{2}\right)\right](x)
$$

which are short-distance quantities and calculable in perturbation theory,

$$
C_{a, i}\left(x, \alpha_{\mathrm{s}}\right)=\left(\delta_{a T} \delta_{i q}+\delta_{a \phi} \delta_{i g}\right) \delta(1-x)+a_{\mathrm{s}} c_{a, i}^{(1)}(x)+a_{\mathrm{s}}^{2} c_{a, i}^{(2)}(x)+\ldots
$$

Here we have identified, again without loss of information, the scale $\mu$ in Eq. (1.1) with the physical scale $Q$, and we have suppressed electroweak charge factors. Besides the quantities in Eq. (1.6), we have included the fragmentation functions $F_{\phi}$ for the exchange of a scalar $\phi$ coupling (like the Higgs-boson in the limit of a heavy top quark and $n_{f}$ massless flavours [3]) directly only to gluons via $\phi G_{\mu \nu}^{a} G_{a}^{\mu \nu}$, where $G_{\mu \nu}^{a}$ denotes the gluon field strength tensor.

The splitting functions (1.4) and coefficient functions (1.8) are known (with a minor caveat in the former case which is not relevant in the present context) to order $\alpha_{s}^{3}$ and $\alpha_{s}^{2}$, respectively, see Refs. [4-9] and references therein. These results provide the next-to-next-to-leading order (NNLO) approximation of (renormalization-group improved) perturbative QCD except for $F_{L}$, where the third-order contributions to Eq. (1.8) would also be needed. All these quantities exhibit, in contrast to their initial-state and DIS counterparts [10-12], a double-logarithmic enhancement at small $x$, i.e., the contributions at order $\alpha_{\mathrm{s}}^{n}$ are enhanced by terms of the form $x^{-1} \ln ^{2 n-a} x$ (the minimal offset $a$ depends on the quantity under consideration) which correspond to poles $\alpha_{\mathrm{s}}^{n} /(N-1)^{2 n+1-a}$ after performing the Mellin transformation (1.3). These terms spoil the convergence of the expansions (1.4) and (1.8) already at $x \lesssim 10^{-2}$ [8, 9] and, obviously, preclude describing particle numbers (multiplicities) given by the first moments, $N=1$, of the fragmentation distributions $D_{p}^{h}$.

At leading and next-to-leading logarithmic (LL and NLL) accuracy, these issues were addressed long ago, see Refs. [13-15], by showing that these small- $x$ contributions can be calculated to all orders. For example, the leading logarithms $(a=2)$ of $P_{\mathrm{gg}}^{T}$ can be resummed to yield

$$
P_{\mathrm{gg}}^{T}\left(N, \alpha_{\mathrm{s}}\right) \stackrel{\mathrm{LL}}{=} \frac{1}{4}(N-1)\left\{\left(1+\frac{32 C_{A} a_{\mathrm{s}}}{(N-1)^{2}}\right)^{1 / 2}-1\right\}
$$

with $C_{A}=n_{\text {colours }}=3$ in QCD, which leads to a contribution proportional to $\sqrt{\alpha_{\mathrm{s}}}$ at $N=1$. More recently there has been renewed interest in the all- $x$ and $N=1$ evolution of (parton) fragmentation functions, see, e.g., Refs. [16-18]. In particular, a new method has been developed in Ref. [19] for carrying out the small- $x$ resummation up to the third logarithms in the standard $\overline{\mathrm{MS}}$ factorization scheme not adopted in Ref. [14] (see Ref. [17] for a more detailed discussion of this issue).

This article builds upon and extends the results of Ref. [19] which were mostly presented in terms of perturbative coefficients to order $\alpha_{\mathrm{s}}^{16}$. While this is sufficient for collider applications 
down to $x \simeq 10^{-4}$, it does neither fix the first moments nor cover the vastly wider $x$-range relevant for fragmentation processes induced by ultra-high energy cosmic rays, see, e.g., Ref. [20]. Here, we provide analytic resummed small- $x$ expressions at next-to-next-to-leading logarithmic (NNLL) accuracy which facilitate an all- $x$ 'NLO + resummed' evolution of the fragmentation functions, and derive the third terms in the resulting $N=1$ expansion in powers of $\sqrt{\alpha_{\mathrm{s}}}$. These results include the case of $F_{L}$, which was dealt with only at NLL level in Ref. [19]. Furthermore we use the approach of Dokshitzer, Marchesini and Salam (DMS) in Ref. [21], which relates the evolution of flavour non-singlet fragmentation and parton distributions, to provide an alternative derivation of the results for the 'non-singlet' part of $P_{\mathrm{gg}}^{T}$ and to extend its resummation to the fifth logarithms and the ${\sqrt{\alpha_{\mathrm{s}}}}^{5}$ contributions at $N=1$ in a manner outlined already in Ref. [22], see also Ref. [23].

\section{Formalism of the resummation}

Before we present our results, we briefly recall the formalism for deriving the resummation, which is based on the mass-factorization relations and the structure of the unfactorized expressions

$$
\widehat{F}_{a, k}=\widetilde{C}_{a, i} \otimes Z_{i k}^{T} \quad \text { for } \quad a=T, \phi, L \quad \text { and } \quad k=\mathrm{q}, \mathrm{g}
$$

in dimensional regularization (we use $D=4-2 \varepsilon$ ). The functions $\widetilde{C}_{a, i}$ are given by Taylor series in $\varepsilon$, with the $\varepsilon^{k}$ terms including $k$ more powers in $\ln x$ than the 4-dimensional coefficient functions (1.8). The transition matrix $Z^{T}$ consist of only negative powers of $\varepsilon$ and can be written in terms of the splitting functions (1.4) and the expansion coefficients $\beta_{n}$ of the beta function of QCD, $\beta\left(\alpha_{\mathrm{s}}\right)=-\beta_{0} a_{\mathrm{s}}^{2}-\ldots$ with $\beta_{0}=\frac{11}{3} C_{A}-\frac{2}{3} n_{f}$. This dependence can be summarized as

$$
a_{\mathrm{s}}^{n} \varepsilon^{-n}: P_{0}^{T}, \beta_{0} ; \quad a_{\mathrm{s}}^{n} \varepsilon^{-n+1}: \ldots+P_{1}^{T}, \beta_{1} ; \ldots ; \quad a_{\mathrm{s}}^{n} \varepsilon^{-1}: P_{n-1}^{T} .
$$

Hence fixed-order knowledge at $\mathrm{N}^{m} \mathrm{LO}$ (i.e., of the splitting functions to $P_{m}$ and the corresponding coefficient functions) fixes the first $m+1$ coefficients in the $\varepsilon$-expansion of $\widehat{F}_{a, k}$ at all orders in $\alpha_{s}$.

The small- $x$ expansions of $\widehat{F}_{T, \phi}$ (the corresponding relation for $F_{L}$ is slightly different) read

$$
\left.\widehat{F}\right|_{a_{\mathrm{s}}^{n} \varepsilon^{-n+\ell}}=\mathcal{F}_{n, \ell}^{(0)} x^{-1} \ln ^{n+\ell-1} x+\mathcal{F}_{n, \ell}^{(1)} x^{-1} \ln ^{n+\ell-2} x+\ldots .
$$

If the constants up to $\mathcal{F}_{n, \ell}^{(m)}$ are known for all $n$ and $\ell$, then the splitting functions and coefficient functions can be determined at $\mathrm{N}^{m} \mathrm{LL}$ accuracy at all orders of the strong coupling. As observed in Ref. [19], the $n^{\text {th }}$ order small- $x$ contributions to $\widehat{F}_{T, \phi}$ are built up from $n$ terms of the form

$$
\left(A_{n, k} \varepsilon^{-2 n+1}+B_{n, k} \varepsilon^{-2 n+2}+\ldots\right) x^{-1-2 k \varepsilon}, \quad k=1, \ldots, n .
$$

Since the terms with $\varepsilon^{-2 n+1}, \ldots, \varepsilon^{-n-1}$ have to cancel in sum (2.1), there are $n-1$ relations between the LL coefficients $A_{n, k}$ which lead to the constants $\mathcal{F}_{n, \ell}^{(0)}$ in Eq. (2.3), $n-2$ relations between the NLL coefficients $B_{n, k}$ etc. As discussed above, a $\mathrm{N}^{m} \mathrm{LO}$ calculation fixes the (nonvanishing) coefficients of $\varepsilon^{-n}, \ldots, \varepsilon^{-n+m}$ at all orders $n$, adding $m+1$ more relations between the coefficients in Eq. (2.4). Consequently the highest $m+1$ double logarithms, i.e., the $\mathrm{N}^{m} \mathrm{LL}$ approximation, can be determined order by order from the $\mathrm{N}^{m} \mathrm{LO}$ results. Finally the resulting series, here calculated to order $\alpha_{\mathrm{s}}^{18}$ using FORM and TFORM [24], can be employed to find their generating functions via over-constrained systems of linear equations. The whole procedure is analogous to, if computationally more involved than, the large- $x$ resummation in Ref. [25]. 


\section{$3 \quad N$-space results: splitting functions $P_{i k}^{T}$}

It turns out that the resummed splitting functions in Mellin space can be expressed in terms of

$$
S=(1-4 \xi)^{1 / 2} \quad \text { and } \quad \mathcal{L}=\ln \left(\frac{1}{2}(1+S)\right)=-\ln \left(\frac{1}{2 \xi}(1-S)\right)
$$

with $\xi=-8 C_{A} a_{\mathrm{s}} / \bar{N}^{2}$ and $\bar{N} \equiv N-1$. At NNLL accuracy, i.e., resumming the contributions $\alpha_{\mathrm{s}}^{n} x^{-1} \ln ^{2 n-a} x$ with $a=2,3,4$ the (flavour-singlet) splitting functions in Eq. (1.1) are given by

$$
\begin{aligned}
P_{\mathrm{qq}}^{T}(N)= & \frac{4}{3} \frac{C_{F} n_{f}}{C_{A}} a_{\mathrm{s}}\left\{\frac{1}{2 \xi}(S-1)(\mathcal{L}+1)+1\right\} \\
+ & \frac{1}{18} \frac{C_{F} n_{f}}{C_{A}^{3}} a_{\mathrm{s}} \bar{N}\left\{\left(-11 C_{A}^{2}+6 C_{A} n_{f}-20 C_{F} n_{f}\right) \frac{1}{2 \xi}(S-1+2 \xi)+10 C_{A}^{2} \frac{1}{\xi}(S-1) \mathcal{L}\right. \\
& -\left(51 C_{A}^{2}-6 C_{A} n_{f}+12 C_{F} n_{f}\right) \frac{1}{2}(S-1)+\left(11 C_{A}^{2}+2 C_{A} n_{f}-4 C_{F} n_{f}\right) S^{-1} \mathcal{L} \\
& \left.+\left(5 C_{A}^{2}-2 C_{A} n_{f}+6 C_{F} n_{f}\right) \frac{1}{\xi}(S-1) \mathcal{L}^{2}+\left(51 C_{A}^{2}-14 C_{A} n_{f}+36 C_{F} n_{f}\right) \mathcal{L}\right\} \\
P_{\mathrm{qg}}^{T}(N)= & \frac{C_{A}}{C_{F}} P_{\mathrm{qq}}^{T}(N)-\frac{2}{9} \frac{n_{f}}{C_{A}^{2}} a_{\mathrm{s}} \bar{N}\left(C_{A}^{2}+C_{A} n_{f}-2 C_{F} n_{f}\right)\left\{\frac{1}{2 \xi}(S-1)(\mathcal{L}+1)+1\right\} \\
P_{\mathrm{gg}}^{T}(N)= & \frac{1}{4} \bar{N}(S-1)-\frac{1}{6 C_{A}} a_{\mathrm{s}}\left(11 C_{A}^{2}+2 C_{A} n_{f}-4 C_{F} n_{f}\right)\left(S^{-1}-1\right)-P_{\mathrm{qq}}^{T}(N) \\
+ & \frac{1}{576 C_{A}^{3}} a_{\mathrm{s}} \bar{N}\left\{\left(\left[1193-576 \zeta_{2}\right] C_{A}^{4}-140 C_{A}^{3} n_{f}+4 C_{A}^{2} n_{f}^{2}-56 C_{A}^{2} C_{F} n_{f}+16 C_{A} C_{F} n_{f}^{2}\right.\right. \\
& \left.-48 C_{F}^{2} n_{f}^{2}\right)(S-1)+\left(\left[830-576 \zeta_{2}\right] C_{A}^{4}+96 C_{A}^{3} n_{f}-8 C_{A}^{2} n_{f}^{2}-208 C_{A}^{2} C_{F} n_{f}\right. \\
& \left.\left.+64 C_{A} C_{F} n_{f}^{2}-96 C_{F}^{2} n_{f}^{2}\right)\left(S^{-1}-1\right)+\left(11 C_{A}^{2}+2 C_{A} n_{f}-4 C_{F} n_{f}\right)^{2}\left(S^{-3}-1\right)\right\} \\
& \frac{C_{F}}{C_{A}} P_{\mathrm{gg}}^{T}(N)-\frac{1}{3} \frac{C_{F}}{C_{A}^{2}} a_{\mathrm{s}}\left(C_{A}^{2}+C_{A} n_{f}-2 C_{F} n_{f}\right) \frac{1}{\xi}\left(S-1+2 \xi^{2}\right) \\
+ & \frac{1}{36} \frac{C_{F}}{C_{A}^{4}} a_{\mathrm{s}} \bar{N}\left\{\left(11 C_{A}^{4}+13 C_{A}^{2} n_{f}\left(C_{A}-2 C_{F}\right)+2 C_{A}^{2} n_{f}^{2}-8\left(C_{A}-C_{F}\right) C_{F} n_{f}^{2}\right)\left(1-S^{-1}\right)\right. \\
& -\left(48 C_{A}^{4}-45 C_{A}^{3} C_{F}-72 \zeta_{2} C_{A}^{3}\left(C_{A}-C_{F}\right)-33 C_{A}^{3} n_{f}+2 C_{A}^{2} n_{f}^{2}+48 C_{A}^{2} C_{F} n_{f}\right. \\
& \left.-8 C_{F}^{2} n_{f}^{2}\right) \frac{1}{\xi}(S-1+2 \xi)+\left(-54 C_{A}^{4}+45 C_{A}^{3} C_{F}+72 \zeta_{2} C_{A}^{3}\left(C_{A}-C_{F}\right)+23 C_{A}^{3} n_{f}\right. \\
& \left.\left.-28 C_{A}^{2} n_{f} C_{F}-8\left(C_{A}-2 C_{F}\right) C_{F} n_{f}^{2}\right) \frac{1}{\xi}(S-1) \mathcal{L}\right\} \\
P_{\mathrm{gq}}^{T}(N) & (3.5) \\
& \\
&
\end{aligned}
$$

with $C_{F}=4 / 3$ in QCD and $\zeta_{2}=\pi^{2} / 6$. The respective first lines of Eqs. (3.2) - (3.5) are the LL (for $P_{\mathrm{gq}}^{T}$ and $P_{\mathrm{gg}}^{T}$, already determined in Ref. [13]) and NLL contributions, the rest represents the NNLL terms. Of course, no negative powers of $C_{A}$ and no non- $(N-1)^{-1}$ terms remain when these results are expanded in powers of $a_{\mathrm{s}}$. After combination with the LO and NLO splitting functions [4] with the $1 /(N-1)^{\ell}, 1 \leq \ell \leq 3$ poles removed to avoid double counting - these results provide a combined ( $N=1$ finite) all- $x$ 'NLO + resummed' evolution of the $\overline{\mathrm{MS}}$ fragmentation distributions. The crucial step towards deriving Eqs. (3.2) - (3.5) is discussed in Appendix A.

As discussed in Ref. [19], it is possible to also obtain the next $\left(\mathrm{N}^{3} \mathrm{LL}\right)$ contributions to $P_{\mathrm{qq}}^{T}$ and $P_{\mathrm{qg}}^{T}$, due to $A_{n, 1}=B_{n, 1}=\ldots=0$ in Eq. (2.4) for $\widehat{F}_{T, \mathrm{q}}$ and $\widehat{F}_{\phi, \mathrm{q}}$. We have been able to find the exact all-order expression also for these contributions. However, the results are considerably more lengthy than Eqs. (3.2) and (3.3) and hence are deferred to Eqs. (B.1) and (B.2) in Appendix B. 
The first moments of the combined splitting functions receive contributions from (3.2) - (3.5), taking the limit $N \rightarrow 1$ for fixed $\alpha_{\mathrm{s}}$, and the 'truncated' fixed-order results $\bar{P}^{T(m)}$ with

$$
\bar{P}_{\mathrm{qq}}^{T(0)}=0, \bar{P}_{\mathrm{qg}}^{T(0)}=\frac{4}{3} n_{f}, \bar{P}_{\mathrm{gq}}^{T(0)}=-3 C_{F}, \bar{P}_{\mathrm{gg}}^{T(0)}=-\frac{11}{3} C_{A}-\frac{2}{3} n_{f}
$$

at $N=1$. This leads to the 'NLO + resummed' results

$$
\begin{aligned}
P_{\mathrm{qg}}^{T}(N=1)= & \frac{8}{3} n_{f} a_{\mathrm{s}}-\frac{1}{3 C_{A}^{2}}\left(17 C_{A}^{2} n_{f}-2 C_{A} n_{f}^{2}+4 C_{F} n_{f}^{2}\right)\left(2 C_{A} a_{\mathrm{s}}^{3}\right)^{1 / 2}+O\left(a_{\mathrm{s}}^{2}\right), \\
P_{\mathrm{qq}}^{T}(N=1)= & \frac{C_{F}}{C_{A}}\left(P_{\mathrm{qg}}^{T}(N=1)-\frac{4}{3} n_{f} a_{\mathrm{s}}\right)+O\left(a_{\mathrm{s}}^{2}\right) \\
P_{\mathrm{gg}}^{T}(N=1)= & \left(2 C_{A} a_{\mathrm{s}}\right)^{1 / 2}-\frac{1}{6 C_{A}}\left(11 C_{A}^{2}+2 C_{A} n_{f}+12 C_{F} n_{f}\right) a_{\mathrm{s}} \\
+ & \frac{1}{144 C_{A}^{3}}\left(\left[1193-576 \zeta_{2}\right] C_{A}^{4}-140 C_{A}^{3} n_{f}+4 C_{A}^{2} n_{f}^{2}+760 C_{A}^{2} C_{F} n_{f}\right. \\
& \left.-80 C_{A} C_{F} n_{f}^{2}+144 C_{F}^{2} n_{f}^{2}\right)\left(2 C_{A} a_{\mathrm{s}}^{3}\right)^{1 / 2}+O\left(a_{\mathrm{s}}^{2}\right) \\
P_{\mathrm{gq}}^{T}(N=1)= & \frac{C_{F}}{C_{A}}\left(P_{\mathrm{gg}}^{T}(N=1)+\frac{4}{3} \frac{C_{F} n_{f}}{C_{A}} a_{\mathrm{s}}\right)+O\left(a_{\mathrm{s}}^{2}\right) .
\end{aligned}
$$

It is interesting to note that the combination $P_{\mathrm{qq}}^{T}-P_{\mathrm{qg}}^{T}+P_{\mathrm{gq}}^{T}-P_{\mathrm{gg}}^{T}$ of the resummed first moments (3.7) vanishes for $C_{A}=C_{F}$ irrespective of the numbers of flavours $n_{f}$. Instead inserting the QCD values for the colour factors, we obtain for $n_{f}=5$ the numerical series

$$
\begin{array}{rr}
P_{\mathrm{qq}}^{T}(N=1) \cong & 0.2358 \alpha_{\mathrm{s}}-0.6773 \alpha_{\mathrm{s}}^{3 / 2}+0.5880 \alpha_{\mathrm{s}}^{2} \\
P_{\mathrm{qg}}^{T}(N=1) \cong & 1.0610 \alpha_{\mathrm{s}}-1.5240 \alpha_{\mathrm{s}}^{3 / 2}+1.8089 \alpha_{\mathrm{s}}^{2} \\
P_{\mathrm{gq}}^{T}(N=1) \cong 0.3071 \alpha_{\mathrm{s}}^{1 / 2}-0.3059 \alpha_{\mathrm{s}}+0.2884 \alpha_{\mathrm{s}}^{3 / 2}, \\
P_{\mathrm{gg}}^{T}(N=1) \cong 0.6910 \alpha_{\mathrm{s}}^{1 / 2}-0.9240 \alpha_{\mathrm{s}}+0.6490 \alpha_{\mathrm{s}}^{3 / 2}
\end{array}
$$

with benign coefficients in terms of a rather large expansion parameter with $\sqrt{\alpha_{\mathrm{s}}} \simeq 0.34$ at $\mu=M_{Z}$. In order to illustrate this behaviour we have included the $\alpha_{\mathrm{s}}^{2}$ coefficients resulting from Eqs. (B.1) and $\left(\overline{\mathrm{B} .2)}\right.$ together with the corresponding truncated second-order splitting functions $\bar{P}_{\mathrm{qi}}^{T(1)}$.

\section{Coefficient functions for $F_{T}$ and $F_{\phi}$}

We now turn to the coefficient functions $(1.8)$ for $F_{T}$ and $F_{\phi}$. Their leading and next-to-leading logarithms, $\alpha_{\mathrm{s}}^{n} x^{-1} \ln ^{2 n-a} x$ with $a=1,2$, need to be included in a 'NLO + resummed' approximation which is applicable at all values of $x$ and finite at $N=1$. The corresponding $N$-space results, again derived from the series expansions in Ref. [19], are given by (using $F \equiv S^{-1 / 2}$ )

$$
\begin{aligned}
C_{T, \mathrm{~g}}(N)= & \frac{C_{F}}{C_{A}}(F-1)+\frac{1}{1152} \frac{C_{F}}{C_{A}^{3}} \bar{N}\left\{\left(-555 C_{A}^{2}+66 C_{A} n_{f}-480 C_{F} n_{f}\right)\left(F^{-1}-1\right)\right. \\
& +\left(868 C_{A}^{2}+152 C_{A} n_{f}-336 C_{F} n_{f}\right)(F-1)+\left(11 C_{A}^{2}-2 C_{A} n_{f}\right)\left[6\left(F^{3}-1\right)\right. \\
& \left.\left.-5\left(F^{7}-1\right)\right]-\left(132 C_{A}^{2}+24\left(C_{A}-2 C_{F}\right) n_{f}\right)\left(F^{5}-1\right)+384 C_{F} n_{f} F \mathcal{L}\right\}, \\
C_{T, \mathrm{q}}(N)= & 1+\frac{1}{3} \frac{C_{F} n_{f}}{C_{A}^{2}} \bar{N}\left(F^{-1}-1-F \mathcal{L}\right)
\end{aligned}
$$


and

$$
\begin{aligned}
C_{\phi, \mathrm{g}}(N)= & 1+\frac{C_{A}}{C_{F}} C_{T, \mathrm{~g}}(N)+\frac{1}{12 C_{A}^{2}} \bar{N}\left\{\left(2 C_{A}^{2}-C_{A} n_{f}+2 C_{F} n_{f}\right)\left(F^{-1}-1\right)\right. \\
& \left.-\left(4 C_{A}^{2}+C_{A} n_{f}-2 C_{F} n_{f}\right)(F-1)\right\} \\
C_{\phi, \mathrm{q}}(N)= & \frac{C_{A}}{C_{F}}\left(C_{T, \mathrm{q}}(N)-1\right) .
\end{aligned}
$$

Here we have included, besides the resummed $1 /(N-1)$ terms, the zeroth-order terms in Eq. (1.8). The leading logarithmic contributions in Eqs. (4.1) and (4.3) have been obtained before, in a completely different manner, in Ref. [17] (where, as in Refs. [5, 7], the normalization of $C_{a, \mathrm{~g}}$ in SIA differs by a factors of two from that adopted in the present article and Ref. [9]). We have also been able to derive closed $N$-space resummations of the NNLL contributions, $\alpha_{\mathrm{s}}^{n} x^{-1} \ln ^{2 n-3} x$, to all four quantities. The corresponding more lengthy expressions can be found in Appendix B.

The first moments of Eqs. (4.1) - (4.4) vanish except for $\alpha_{\mathrm{s}}^{0}$ contributions in Eqs. (4.1) and (4.2). Note, in particular, that the zeroth-order contribution (1.8) is compensated in $C_{\phi, g}$ by the resummation of the leading logarithms. The first $\alpha_{\mathrm{s}}$-dependent contributions are due to Eqs. (B.7) - (B.10) and the truncated NLO coefficient functions $\bar{c}_{a, i}^{(1)}$ (i.e., the corresponding quantities of Eq. (1.8) with the $1 /(N-1)$ poles removed) and read

$$
\begin{aligned}
C_{T, \mathrm{q}}(N=1) & =-\frac{C_{A}}{C_{F}} C_{T, \mathrm{~g}}(N=1)+\ldots \\
& =1+\frac{1}{9} \frac{C_{F}}{C_{A}^{3}}\left(9 C_{A}^{3}+50 C_{A}^{2} n_{f}-8\left(C_{A}-2 C_{F}\right) n_{f}^{2}\right) a_{\mathrm{s}}+\ldots, \\
C_{\phi, \mathrm{q}}(N=1) & =-\frac{C_{A}}{C_{F}} C_{\phi, \mathrm{g}}(N=1)+\ldots=-\frac{4}{9} \frac{n_{f}^{2}}{C_{A}^{2}}\left(C_{A}-2 C_{F}\right) a_{\mathrm{s}}+\ldots .
\end{aligned}
$$

The next corrections at $N=1$ may be expected at order $\alpha_{\mathrm{s}}^{2}$ which is beyond our present reach.

All our results above are given in the standard $\overline{\mathrm{MS}}$ factorization scheme. The transformation to another scheme $S$ is given by

$$
C_{S}(N)=C(N)\left[Z_{S}(N)\right]^{-1}, \quad P_{S}(N)=\left(Z_{S}(N) P(N)+\beta \frac{\partial Z_{S}(N)}{\partial a_{\mathrm{s}}}\right)\left[Z_{S}(N)\right]^{-1},
$$

where (suppressing the Mellin variable and the scheme index) $C$ and $P$ represent the matrices

$$
C=\left(\begin{array}{cc}
C_{T, \mathrm{q}} & C_{T, \mathrm{~g}} \\
C_{\phi, \mathrm{q}} & C_{\phi, \mathrm{g}}
\end{array}\right) \text { and } P=\left(\begin{array}{cc}
P_{\mathrm{qq}}^{T} & P_{\mathrm{gq}}^{T} \\
P_{\mathrm{qg}}^{T} & P_{\mathrm{gg}}^{T}
\end{array}\right) \text {, with } P_{\mathrm{LL}}=\left(\begin{array}{cc}
0 & \frac{C_{F}}{C_{A}} p_{\mathrm{LL}} \\
0 & p_{\mathrm{LL}}
\end{array}\right)
$$

in $\overline{\mathrm{MS}}$. Eq. (4.7) includes, for $Z_{S}=C$, the transformation to the 'physical evolution kernels' $K$,

$$
\frac{\partial}{\partial \ln Q^{2}}\left(\begin{array}{c}
F_{T} \\
F_{\phi}
\end{array}\right)=\left(\begin{array}{cc}
K_{T T} & K_{T \phi} \\
K_{\phi T} & K_{\phi \phi}
\end{array}\right)\left(\begin{array}{c}
F_{T} \\
F_{\phi}
\end{array}\right),
$$

for the system $F=\left(F_{T}, F_{\phi}\right)$ of fragmentation functions [for $N \neq 1$, since $\operatorname{det} C$ vanishes for the results in Eqs. (4.5) and (4.6)], cf. Ref. [26]. At LL accuracy this transformation, as well as that to the massive-gluon scheme of Ref. [14], see also Ref. [17], is of the form

$$
Z_{S, \mathrm{LL}}=\frac{1}{1+c_{S, \mathrm{LL}}}\left(\begin{array}{cc}
1+c_{S, \mathrm{LL}} & \frac{C_{F}}{C_{A}}\left(c_{\mathrm{LL}}-c_{S, \mathrm{LL}}\right) \\
0 & 1+c_{\mathrm{LL}}
\end{array}\right)
$$


with $c_{\mathrm{LL}}=F-1$ [recall $F=S^{-1 / 2}$ with $S$ given in Eq. (3.1)], $c_{S, \mathrm{LL}}=\frac{1}{2}\left(S^{-1}-1\right)$ for the scheme of Ref. [14] and $c_{S . \mathrm{LL}}=0$ for the physical kernels. $P_{\mathrm{LL}}$ is invariant for this form of $Z_{S}$. Since we do not know the coefficient functions in the massive gluon scheme beyond LL accuracy, we are not able to use the scheme transformation to compare our results to the NLL splitting functions in Ref. [14]. The transformation to the physical kernels in Eq. (4.9), on the other hand, does not pose problems. It is interesting to note that, disregarding overall prefactors, the results to NNLL (for $K_{\phi T}$ and $K_{\phi \phi}$ ) and $\mathrm{N}^{3} \mathrm{LL}$ (for $K_{T T}$ and $K_{T \phi}$ ) accuracy include only integer coefficients in the expansion in powers of $\alpha_{s}$. Consequently terms with the logarithm $\mathcal{L}$ in Eq. (3.1) do not enter their closed all-order expressions, in contrast to Eqs. (3.2) - (3.5) for the $\overline{\mathrm{MS}}$ splitting functions.

\section{Coefficient functions for $F_{L}$}

The remaining SIA coefficient functions with $x^{-1} \ln ^{k} x$ contributions are those for the longitudinal fragmentation function $F_{L}$. There is no $\alpha_{\mathrm{s}}^{0}$ term in this case, hence the $\alpha_{\mathrm{s}}^{n}$ results are required for the $\mathrm{N}^{n-1} \mathrm{LO}$ approximation and thus for resummation of the $n$ highest logarithms outlined above. Since the NLO coefficient function $C_{L, \mathrm{~g}}$ includes terms up to $x^{-1} \ln ^{2} x$, the NNLL resummation is required to extend the all- $x$ 'NLO + resummed' approximation to $F_{L}$. The coefficient functions for $F_{L}$ have been calculated so far only to order $\alpha_{\mathrm{s}}^{2}$ [5,7]. It is, however, possible to derive (at least) their highest three $\alpha_{\mathrm{s}}^{3} x^{-1} \ln ^{k} x$ contributions using $x \rightarrow 1 / x$ analytic-continuation $(\mathcal{A C}$ ) relations between DIS and SIA physical kernels along the lines of Ref. [9], see also Ref. [27].

For this purpose we have calculated the evolution kernels $K^{T}$ and $K^{S}$ corresponding to Eq. (4.9) for the system $\left(F_{T}, \widetilde{F}_{L}\right)$ with $\widetilde{F}_{L}\left(N, Q^{2}\right)=F_{L}\left(N, Q^{2}\right) /\left(\alpha_{\mathrm{s}} c_{L, \mathrm{q}}^{(1)}(N)\right)$ and its 'spacelike' counterpart of DIS structure functions $\left(F_{1}, \widetilde{F}_{L}^{S}\right)$ for which all NNLO coefficient functions are known [10, 11, 28]. At this order these kernels are expected to be related by

$$
K_{a b}^{T}\left(x, \alpha_{\mathrm{s}}\right)=\mathcal{A C}\left[K_{a b}^{S}\left(x, \alpha_{\mathrm{s}}\right)\right]
$$

up to terms beyond the presently required logarithmic accuracy. These relations form a check of the respective two highest logarithms in $c_{L, \mathrm{q}}^{(3)}$ and $c_{L, \mathrm{~g}}^{(3)}$, which have been derived by resumming the NLO results in Ref. [19], and provide an over-constrained system of four equations for the hitherto unknown coefficients of $\alpha_{\mathrm{s}}^{3} x^{-1} \ln ^{2} x$ in $C_{L, \mathrm{~g}}$ and $\alpha_{\mathrm{s}}^{3} x^{-1} \ln x$ in $C_{L, \mathrm{q}}$. Eq. (5.1) leads to

$$
\begin{aligned}
c_{L, \mathrm{q}}^{(3)}(N)= & 256 C_{A} C_{F} n_{f} \bar{N}^{-4}-64 C_{F}\left(\frac{17}{3} C_{A} n_{f}-\frac{2}{3} C_{F} n_{f}-\frac{4}{9} n_{f}^{2}\right) \bar{N}^{-3} \\
& -64 C_{F}\left(\frac{94}{27} C_{A} n_{f}-\left[\frac{5}{6}-\frac{4}{3} \zeta_{2}\right] C_{F} n_{f}-\frac{1}{3} n_{f}^{2}\right) \bar{N}^{-2}+O\left(\bar{N}^{-1}\right), \\
c_{L, \mathrm{~g}}^{(3)}(N)= & 1408 C_{A}^{2} C_{F} \bar{N}^{-5}-32 C_{F}\left(\frac{241}{3} C_{A}^{2}-10 C_{A} C_{F}-\frac{20}{3} C_{F} n_{f}-\frac{2}{3} C_{A} n_{f}\right) \bar{N}^{-4} \\
& +32 C_{F}\left(\left[19-22 \zeta_{2}\right] C_{A}^{2}-\frac{17}{6} C_{A} C_{F}+\frac{61}{18} C_{A} n_{f}-\frac{37}{9} C_{F} n_{f}\right) \bar{N}^{-3}+O\left(\bar{N}^{-2}\right) .
\end{aligned}
$$

The resulting NNLL resummation for $F_{L}$ - which does not involve Eq. (5.2) - can be written as

$$
C_{L, \mathrm{q}}(N)=\frac{2}{3} \frac{C_{F} n_{f}}{C_{A}} a_{\mathrm{s}}\left\{\frac{1}{\xi}(S-1)\left(F \mathcal{L}-F^{-1}\right)-2\right\}
$$




$$
\begin{aligned}
& +\frac{1}{1728} \frac{C_{F} n_{f}}{C_{A}^{3}} a_{\mathrm{s}} \bar{N}\left\{96\left(5 C_{A}^{2}-2 C_{A} n_{f}+6 C_{F} n_{f}\right) \frac{1}{\xi}(S-1) F \mathcal{L}^{2}-\left(231 C_{A}^{2}-42 C_{A} n_{f}\right.\right. \\
& \left.-32 C_{A} C_{F}+128 C_{F} n_{f}\right) 9 \frac{1}{\xi}(S-1) F^{-1} \mathcal{L}+8\left(53 C_{A}^{2}+28 C_{A} n_{f}+36 C_{A} C_{F}\right. \\
& \left.-114 C_{F} n_{f}\right) \frac{1}{\xi}(S-1) F \mathcal{L}-6\left(55 C_{A}^{2}+6 C_{A} n_{f}-16 C_{F} n_{f}\right) \frac{1}{\xi}(S-1) F^{3} \mathcal{L} \\
& -24\left(11 C_{A}^{2}-2 C_{F} n_{f}\right) \frac{1}{\xi}(S-1) F^{5} \mathcal{L}-5\left(11 C_{A}^{2}-2 C_{A} n_{f}\right)\left(\frac{1}{\xi}(S-1) F^{7} \mathcal{L}\right. \\
& \left.+4\left(F^{5}-1\right)\right)-16\left(71 C_{A}^{2}+10 C_{A} n_{f}-48 C_{F} n_{f}\right) \frac{1}{\xi}\left((S-1) F^{-1}+2 \xi\right) \\
& -12\left(577 C_{A}^{2}-54 C_{A} n_{f}-96 C_{F} C_{A}+144 C_{F} n_{f}\right)\left(F^{-1}-1\right)+4\left(755 C_{A}^{2}\right. \\
& \left.\left.-146 C_{A} n_{f}+384 C_{F} n_{f}\right)(F-1)-4\left(209 C_{A}^{2}+10 C_{A} n_{f}-48 C_{F} n_{f}\right)\left(F^{3}-1\right)\right\}, \\
& C_{L, \mathrm{~g}}(N)=\frac{1}{4} \frac{C_{F}}{C_{A}} \bar{N}\left(F^{-1}-F\right)-\frac{C_{F}}{C_{A}} c_{L \mathrm{q}}^{T}(N) \\
& +\frac{1}{144} \frac{C_{F}}{C_{A}^{2}} a_{\mathrm{s}}\left\{16\left(29 C_{A}^{2}+C_{A} n_{f}-3 C_{F} n_{f}\right)\left(\frac{1}{\xi}(S-1) F^{-1}+2\right)+\left(737 C_{A}^{2}+58 C_{A} n_{f}\right.\right. \\
& \left.+288 C_{A} C_{F}-192 C_{F} n_{f}\right)(F-1)-\left(121 C_{A}^{2}+26 C_{A} n_{f}-48 C_{F} n_{f}\right)\left(F^{3}-1\right) \\
& \left.-\left(209 C_{A}^{2}+10 C_{A} n_{f}-48 C_{F} n_{f}\right)\left(F^{5}-1\right)-5\left(11 C_{A}^{2}-2 C_{A} n_{f}\right)\left(F^{7}-1\right)\right\} \\
& +\frac{1}{331776} \frac{C_{F}}{C_{A}^{4}} a_{\mathrm{s}} \bar{N}\left\{-256\left(\left[104-4212 \zeta_{2}\right] C_{A}^{4}-459 C_{A}^{3} C_{F}+533 C_{A}^{3} n_{f}-480 C_{A}^{2} C_{F} n_{f}\right.\right. \\
& \left.-16 C_{A}^{2} n_{f}^{2}-60 C_{F} n_{f}^{2}\left[C_{A}-3 C_{F}\right]\right)\left(\frac{1}{\xi}(S-1) F^{-1}+2\right)-36864 C_{F} n_{f}\left(C_{A}^{2}+C_{A} n_{f}\right. \\
& \left.-2 C_{F} n_{f}\right)\left(\frac{1}{\xi}(S-1) F \mathcal{L}\right)+\left(\left[221217+101376 \zeta_{2}\right] C_{A}^{4}-77760 C_{A}^{3} C_{F}-3852 C_{A}^{3} n_{f}\right. \\
& \left.-9856 C_{A}^{2} C_{F} n_{f}-512\left[12 C_{A} C_{F}^{2} n_{f}+C_{A} C_{F} n_{f}^{2}-3 C_{F}^{2} n_{f}^{2}\right]-92 C_{A}^{2} n_{f}^{2}\right) 9\left(F^{-1}-1\right) \\
& -\left(\left[100985-1575936 \zeta_{2}\right] C_{A}^{4}-451584 C_{A}^{3} C_{F}+234740 C_{A}^{3} n_{f}-160032 C_{A}^{2} C_{F} n_{f}\right. \\
& \left.+193536 C_{A} C_{F}^{2} n_{f}-14524 C_{A}^{2} n_{f}^{2}+87744 C_{A} C_{F} n_{f}^{2}-124416 C_{F}^{2} n_{f}^{2}\right)(F-1) \\
& -3\left(116329 C_{A}^{4}+55296 \zeta_{2} C_{A}^{4}+23668 C_{A}^{3} n_{f}+1924 C_{A}^{2} n_{f}^{2}-12672 C_{A}^{3} C_{F}\right. \\
& \left.-55776 C_{A}^{2} C_{F} n_{f}-12480 C_{A} C_{F} n_{f}^{2}+16128 C_{F}^{2} n_{f}^{2}\right)\left(F^{3}-1\right)-3\left(126471 C_{A}^{4}\right. \\
& +55296 \zeta_{2} C_{A}^{4}+380 C_{A}^{2} n_{f}\left(5 C_{A}+n_{f}\right)+25344 C_{A}^{3} C_{F}-50752 C_{A}^{2} C_{F} n_{f} \\
& \left.-9344 C_{A} C_{F} n_{f}^{2}-9216 C_{A} C_{F}^{2} n_{f}+17664 C_{F}^{2} n_{f}^{2}\right)\left(F^{5}-1\right)-3\left(71511 C_{A}^{4}\right. \\
& -18260 C_{A}^{3} n_{f}-1348 C_{A}^{2} n_{f}^{2}+10560 C_{A}^{3} C_{F}-9664 C_{A}^{2} C_{F} n_{f}+6016 C_{A} C_{F} n_{f}^{2} \\
& \left.-2304 C_{F}^{2} n_{f}^{2}\right)\left(F^{7}-1\right)+\left(123541 C_{A}^{4}+55396 C_{A}^{3} n_{f}-2636 C_{A}^{2} n_{f}^{2}-\left(10 C_{A} C_{F} n_{f}^{2}\right.\right. \\
& \left.\left.+209 C_{A}^{2} C_{F} n_{f}\right) 480+11520 C_{F}^{2} n_{f}^{2}\right)\left(F^{9}-1\right)+\left(4477 C_{A}^{4}-572 C_{A}^{3} n_{f}-44 C_{A}^{2} n_{f}^{2}\right. \\
& \left.\left.-96 C_{A} C_{F} n_{f}\left(11 C_{A}-2 n_{f}\right)\right) 35\left(F^{11}-1\right)+385 C_{A}^{2}\left(11 C_{A}-2 n_{f}\right)^{2}\left(F^{13}-1\right)\right\} .
\end{aligned}
$$

Eqs. (5.4) and (5.5) complete the results required for the 'NLO + resummed' all- $x$ flavour-singlet evolution of the fragmentation distributions (1.1) and fragmentation functions (1.6). It is interesting to note that, unlike the corresponding splitting functions and kernels for the system $\left(F_{T}, F_{\phi}\right)$, the upper-row elements $K_{T a}$ of the resummed evolution matrix for $\left(F_{T}, \widetilde{F}_{L}\right)$ are suppressed by two powers of $\bar{N}=N-1$ with respect to their lower-row counterparts $K_{L a}$. Also these kernels are found to include only integer coefficients after expansion in powers of $a_{\mathrm{s}}=\alpha_{\mathrm{s}} /(4 \pi)$ or $\alpha_{\mathrm{s}} / \pi$. 


\section{DMS relation and 'non-singlet' resummation of $P_{\mathrm{gg}}^{T}$}

Another connection between the final-state ('timelike') and initial-state ('spacelike') evolution has been suggested in Ref. [21]. The evolution of the flavour non-singlet fragmentation distributions and parton distributions, respectively denoted as $f_{1}$ and $f_{-1}$, is written as

$$
\frac{\partial}{\partial \ln Q^{2}} f_{\sigma}\left(x, Q^{2}\right)=\left[P_{u}\left(\alpha_{\mathrm{s}}\left(Q^{2}\right)\right) \otimes f_{\sigma}\left(z^{\sigma} Q^{2}\right)\right](x)
$$

(the convolution $\otimes$ has been defined in Eq. (1.2) above), and the modified splitting functions $P_{u}$ are postulated to be identical for the timelike and spacelike cases. Eq. (6.1) has been applied and verified for the NNLO non-singlet [6] and gluon-gluon [8] splitting functions. In the latter case its applicability is obvious for Quantum Gluodynamics, $n_{f}=0$, but it is found to hold for all terms in the limit $C_{F}=0$. In $N$-space Eq. (6.1) leads to [21]

$$
\partial_{\ln Q^{2}} f_{\sigma}\left(N, Q^{2}\right)=P_{\sigma}(N) f_{\sigma}\left(N, Q^{2}\right)=P_{u}\left(N+\sigma \partial_{\ln Q^{2}}\right) f_{\sigma}\left(N, Q^{2}\right) .
$$

The solution of this equation, obtained by expanding in powers of $\sigma$, can be cast in the (new) form

$$
P_{\sigma}(N)=P_{u}(N)+\sum_{n=1}^{\infty} \frac{\sigma^{n}}{n !} \frac{\partial^{n-1}}{\partial N^{n-1}}\left(\frac{\partial P_{u}}{\partial N}\left[P_{u}(N)\right]^{n}\right)
$$

suitable for all-order considerations. The above relations imply that the difference $\delta P=P_{1}-P_{-1}$ of the time- and spacelike splitting functions at any order is given by lower-order quantities.

The crucial point in the present context is that the spacelike splitting functions are only singlelogarithmically enhanced with the (scheme-independent) leading terms [29, 30]

$$
P_{\mathrm{gg}}^{S}(N)=\frac{C_{A} \alpha_{\mathrm{s}}}{\pi} \bar{N}^{-1}+2 \zeta_{3}\left(\frac{C_{A} \alpha_{\mathrm{s}}}{\pi}\right)^{4} \bar{N}^{-4}+O\left(\alpha_{\mathrm{s}}^{6} \bar{N}^{-6}\right) \text {. }
$$

Therefore the above differences and the timelike splitting functions are equal, $\delta P_{\mathrm{gg}}^{(n)}=P_{\mathrm{gg}}^{T(n)}$, for $n \geq \ell+1$ at the level of the $\mathrm{N}^{\ell}$ LL small- $x$ double logarithms. The LL, NLL and NNLL contributions to $P_{\mathrm{gg}}^{T(n)}$ for $C_{F}=0$ are thus fixed by Eq. (6.1) to all orders in $\alpha_{\mathrm{s}}$ without any spacelike input beyond the NNLO splitting functions [12], and are found to be identical to the results presented in Eq. (3.4). As already discussed in Ref. [22], the $N^{3} L L$ terms are fixed if Eq. (6.4) is used in addition, and finally the $\mathrm{N}^{4} \mathrm{LL}$ corrections require only one additional coefficient, that of $\alpha_{\mathrm{s}}^{4} \bar{N}^{-3}$, of $P_{\mathrm{gg}}^{S}(N)$. However, this coefficient is presently not available in the literature for the $\overline{\mathrm{MS}}$ scheme.

The resulting prediction for the $\mathrm{N}^{3} \mathrm{LL}$ contribution to $P_{\mathrm{gg}}^{T}$ reads

$$
\begin{aligned}
P_{\mathrm{gg}}^{T}(N) & \left.\right|_{\mathrm{N}^{3} \mathrm{LL}} ^{C_{F}=0}=\frac{1}{13824} \frac{1}{C_{A}} a_{\mathrm{s}}^{2}\left\{4 \left(\left[48125-3168 \zeta_{2}-15552 \zeta_{3}\right] C_{A}^{3}-\left[46+2880 \zeta_{2}\right] C_{A}^{2} n_{f}\right.\right. \\
& \left.+236 C_{A} n_{f}^{2}-8 n_{f}^{3}\right) \frac{1}{\xi}(S-1+2 \xi)-\left(\left[48473+9504 \zeta_{2}-25920 \zeta_{3}\right] C_{A}^{3}-340 C_{A} n_{f}^{2}\right. \\
& \left.+24 n_{f}^{3}-\left[2670-4032 \zeta_{2}\right] C_{A}^{2} n_{f}\right) \frac{1}{\xi^{2}}\left(S-1+2 \xi+2 \xi^{2}\right)+2\left(\left[20337+7920 \zeta_{2}\right.\right. \\
& \left.\left.-12960 \zeta_{3}\right] C_{A}^{3}-\left[2330-2592 \zeta_{2}\right] C_{A}^{2} n_{f}-156 C_{A} n_{f}^{2}+24 n_{f}^{3}\right) \frac{1}{\xi^{2}}\left(S^{-1}-1-2 \xi-6 \xi^{2}\right) \\
& +4\left(\left[1617-1584 \zeta_{2}\right] C_{A}^{3}+\left[316-288 \zeta_{2}\right] C_{A}^{2} n_{f}-40 C_{A} n_{f}^{2}-8 n_{f}^{3}\right) \frac{1}{\xi^{2}}\left(S^{-3}-1\right. \\
& \left.\left.-6 \xi-30 \xi^{2}\right)+\left(11 C_{A}+2 n_{f}\right)^{3} \frac{1}{\xi^{2}}\left(S^{-5}-1-10 \xi-70 \xi^{2}\right)\right\} .
\end{aligned}
$$


Combined with the first moment of the truncated NLO splitting function,

$$
\bar{P}_{\mathrm{gg}}^{T(1)}(N=1)=\frac{160}{27} C_{A}^{2}+\left(\frac{76}{27}+\frac{16}{3} \zeta_{2}\right) C_{A} n_{f}-\frac{88}{3} C_{F} n_{f},
$$

this results yields

$$
\left.P_{\mathrm{gg}}^{T}(N=1)\right|_{a_{\mathrm{s}}^{2}} ^{C_{F}=0}=-\left(\frac{35}{3}-\frac{11}{3} \zeta_{2}-6 \zeta_{3}\right) C_{A}^{2}-\left(\frac{11}{9}-\frac{10}{3} \zeta_{2}\right) C_{A} n_{f}
$$

Including also the $\mathrm{N}^{4} \mathrm{LL}$ contribution in Eq. (B.12), the numerical result for $n_{f}=5$ is given by

$$
\begin{aligned}
\left.P_{\mathrm{gg}}^{T}(N=1)\right|^{C_{F}=0} \cong & 0.6910 \alpha_{\mathrm{s}}^{1 / 2}-0.5703 \alpha_{\mathrm{s}}+0.0267 \alpha_{\mathrm{s}}^{3 / 2}+0.4946 \alpha_{\mathrm{s}}^{2} \\
& +\left(1.0036+\left.0.15753 B_{\mathrm{gg}}^{S(3)}\right|_{\mathrm{NLL}} ^{C_{F}=0}\right) \alpha_{\mathrm{s}}^{5 / 2}+O\left(\alpha_{\mathrm{s}}^{3}\right)
\end{aligned}
$$

where $\left.B_{\mathrm{gg}}^{S(3)}\right|_{\mathrm{NLL}}$ is the above-mentioned next-to-leading small- $x$ coefficient at order $\alpha_{\mathrm{s}}^{4}$, cf. Eq. (1.4), in the notation of Eq. (6.4), i.e., the coefficient of $\left(C_{A} \alpha_{\mathrm{S}} / \pi\right)^{4} \bar{N}^{-3}$. One may expect it to be negative and no larger than a few times the corresponding LL coefficient $2 \zeta_{3} \simeq 2.4041$. An explicit determination of this coefficient and its fifth-order counterpart from the results of Ref. [31] should definitely be possible, in particular in view of the calculations performed in Refs. [32, 33]. On top of these quantities, and the known NNLO splitting function [8] and sixth-order coefficient in Eq. (6.4), the $\alpha_{\mathrm{s}}^{3}$ coefficient in Eq. (6.8) requires the NNLL fourth-order term in Eq. (6.4), as already discussed in Ref. [22], which currently appears to be out of reach.

\section{$7 \quad$ Analytic and numerical results in $x$-space}

We finally return to the now complete 'NLO + resummed' approximations for the $\overline{\mathrm{MS}}$ splitting functions and coefficient functions in Eqs. (1.1) and (1.7). Their numerical consequences in $x$-space can be obtained either by expanding the above $N$-space results to any desired order in $\alpha_{\mathrm{s}}$ and using

$$
\int_{0}^{1} d x x^{N-2} \ln ^{k} x=(-1)^{k} \frac{k !}{(N-1)^{k+1}},
$$

or by subjecting the $N$-space expression to a standard numerical Mellin inversion, cf, e.g., Ref. [34]. The former can also be used to identify the closed form of $x$-space results such as

$$
\begin{aligned}
& x P_{\mathrm{gg}}^{T}\left(x, \alpha_{\mathrm{s}}\right)+\left.x P_{\mathrm{qq}}^{T}\left(x, \alpha_{\mathrm{s}}\right)\right|_{\mathrm{NNLL}}=\left\{4 C_{A} a_{\mathrm{s}}+\frac{8}{3}\left(11 C_{A}^{2}+2 C_{A} n_{f}-4 C_{F} n_{f}\right) a_{\mathrm{s}}^{2} \ln \frac{1}{x}\right\} \frac{2}{z} J_{1}(z) \\
& \quad+\left\{\frac{4}{9}\left(26 C_{F} n_{f}-23 C_{A} n_{f}\right) a_{\mathrm{s}}^{2}+\frac{8}{9 C_{A}}\left(11 C_{A}^{2}+2 C_{A} n_{f}-4 C_{F} n_{f}\right)^{2} a_{\mathrm{s}}^{3} \ln ^{2} \frac{1}{x}\right\} \frac{2}{z} J_{1}(z) \\
& \quad+\frac{32}{9 C_{A}}\left(\left[134-72 \zeta_{2}\right] C_{A}^{4}+23 C_{A}^{3} n_{f}-48 C_{A}^{2} C_{F} n_{f}+4 C_{A} C_{F} n_{f}^{2}-8 C_{F}^{2} n_{f}^{2}\right) a_{\mathrm{s}}^{3} \ln ^{2} \frac{1}{x} \frac{4}{z^{2}} J_{2}(z)
\end{aligned}
$$

and

$$
x P_{\mathrm{gq}}^{T}(N)-\left.\frac{C_{F}}{C_{A}} x P_{\mathrm{gg}}^{T}\left(x, \alpha_{\mathrm{s}}\right)\right|_{\mathrm{NLL}}=-\frac{32}{3} \frac{C_{F}}{C_{A}}\left(C_{A}^{2}+C_{A} n_{f}-2 C_{F} n_{f}\right) a_{\mathrm{s}}^{2} \ln \frac{1}{x} \frac{4}{z^{2}} J_{2}(z)
$$


with

$$
z=\left(32 C_{A} a_{\mathrm{s}}\right)^{1 / 2} \ln \frac{1}{x}
$$

in terms of the Bessel function of the first kind $J_{1}(z)$ and $J_{2}(z)$, cf. Ref. [35]. The first line of Eq. (7.2) represents the LL and NLL terms, and the next two lines the NNLL contribution. The missing ingredients for a completely analytical Mellin (Laplace) inversion of Eqs. (3.2) - (3.5) are the inverses of products of powers of the square root $S$ and the logarithm $\mathcal{L}$ defined in Eq. (3.1). Both this logarithm, which also corresponds to a Bessel function in $x$-space (see Eq. (A.3) below), and the fourth $\operatorname{root} F$ in Eq. (4.1), which corresponds to a hypergeometric function [17], can be viewed as 'artifacts' of the $\overline{\mathrm{MS}}$ scheme, as neither remains after transformation to the matrix of physical evolution kernels (4.9) for the fragmentation functions $F_{T}$ and $F_{\phi}$ and their counterparts for the system $\left(F_{T}, \widetilde{F}_{L}\right)$ discussed above Eq. (5.1).

The result (7.2) for $P_{\mathrm{gg}}^{T}$ indicates a general single-logarithmic enhancement of the Besselfunction oscillations at extremely small $x$, where furthermore the contribution of $J_{2}(z)$ is suppressed by a factor $2 / z$ with, e.g., $z \simeq 0.96 \ln \frac{1}{x}$ for $\alpha_{\mathrm{s}} \simeq 0.12$ - recall that $J_{1}(z)$ and $J_{2}(z)$ differ for large $z$ only by a phase shift of $\pi / 2$ [35]. The asymptotically dominant $a_{\mathrm{s}}\left(a_{\mathrm{s}} \ln \frac{1}{x}\right)^{\ell} \frac{2}{z} J_{1}(z)$ terms arise from the $S^{-2 \ell+1}$ contributions to the $\mathrm{N}^{\ell} \mathrm{LL}$ terms and have closely related prefactors proportional to $\left(11 C_{A}^{2}-2 C_{A} n_{f}+4 C_{F} n_{f}\right)^{\ell}$, see Eqs. (3.4), (6.5) and (B.11), which seem to point towards the possibility of a 'second resummation'. Taking into account the asymptotic $z^{-1 / 2}$ suppression of $J_{n}(z)$, the oscillation amplitudes of these contributions to the LL, NLL and NNLL results behave as $\left(\ln \frac{1}{x}\right)^{-3 / 2},\left(\ln \frac{1}{x}\right)^{-1 / 2}$ and $\left(\ln \frac{1}{x}\right)^{1 / 2}$, respectively.

The LO + LL and NLO + NNLL approximations, which provide the minimal $N=1$ finite resummations of the respective fixed-order results, are illustrated in Fig. 1 for the second column and in Fig. 2 for the first column of the splitting-function matrix $P$ in Eq. (4.8). Note that the scales of the right and left parts of both figures are related by a factor $C_{A} / C_{F}=9 / 4$. The close similarity of the curves at small $x$ thus directly demonstrates the approximate 'Casimir scaling' of $P_{\mathrm{gi}}^{T}$ and $P_{\mathrm{qi}}^{T}$. The oscillation amplitudes of the former quantities are much larger than those of the latter. This behaviour is not due to the LL contributions to $P_{\mathrm{gi}}^{T}$ which, as for other resummations, are numerically small. There is no reason to assume that terms beyond the present NNLL accuracy will be small. The Mellin inversion of Eqs. (6.5) and (B.11), however, appears to indicate that the amplitudes will stabilize, for most of the wide $x$-range shown in the figures, after including the $\mathrm{N}^{3} \mathrm{LL}$ and $\mathrm{N}^{4} \mathrm{LL}$ contributions.

Corresponding minimal 'LO + resummed' and 'NLO + resummed' $x$-space results are shown in Figs. 3 and 4 for the coefficient functions for $F_{T}$ (left) and $F_{L}$ (right). In this counting, the leading contribution $C_{F} / C_{A} c_{\mathrm{LL}}$ to $C_{T, \mathrm{~g}}$ in Eq. (4.1) is not combined with the $\delta(1-x) \mathrm{LO}$ term in Eq. (1.8). This is consistent with the fact that $c_{\mathrm{LL}}$ is a scheme-dependent quantity as discussed around Eq. (4.10). Given the $\alpha_{\mathrm{s}}$-expanded $\mathrm{N}^{3} \mathrm{LL}$ result in Ref. [19], we expect that the coefficient function $C_{T, \mathrm{q}}$, for which Fig. 4 includes the Mellin inverse of Eq. (4.2), will be the first quantity determined at an $N=1$ finite 'NNLO + resummed' accuracy. The opposite is true for $C_{L, \mathrm{~g}}$, the least stable quantity at the present accuracy as shown in the right part of Fig. 3. Here, as for $P_{\text {gi }}^{T}$, the highest five logarithms need to be resummed for an all- $x$ combination with the NNLO results. 

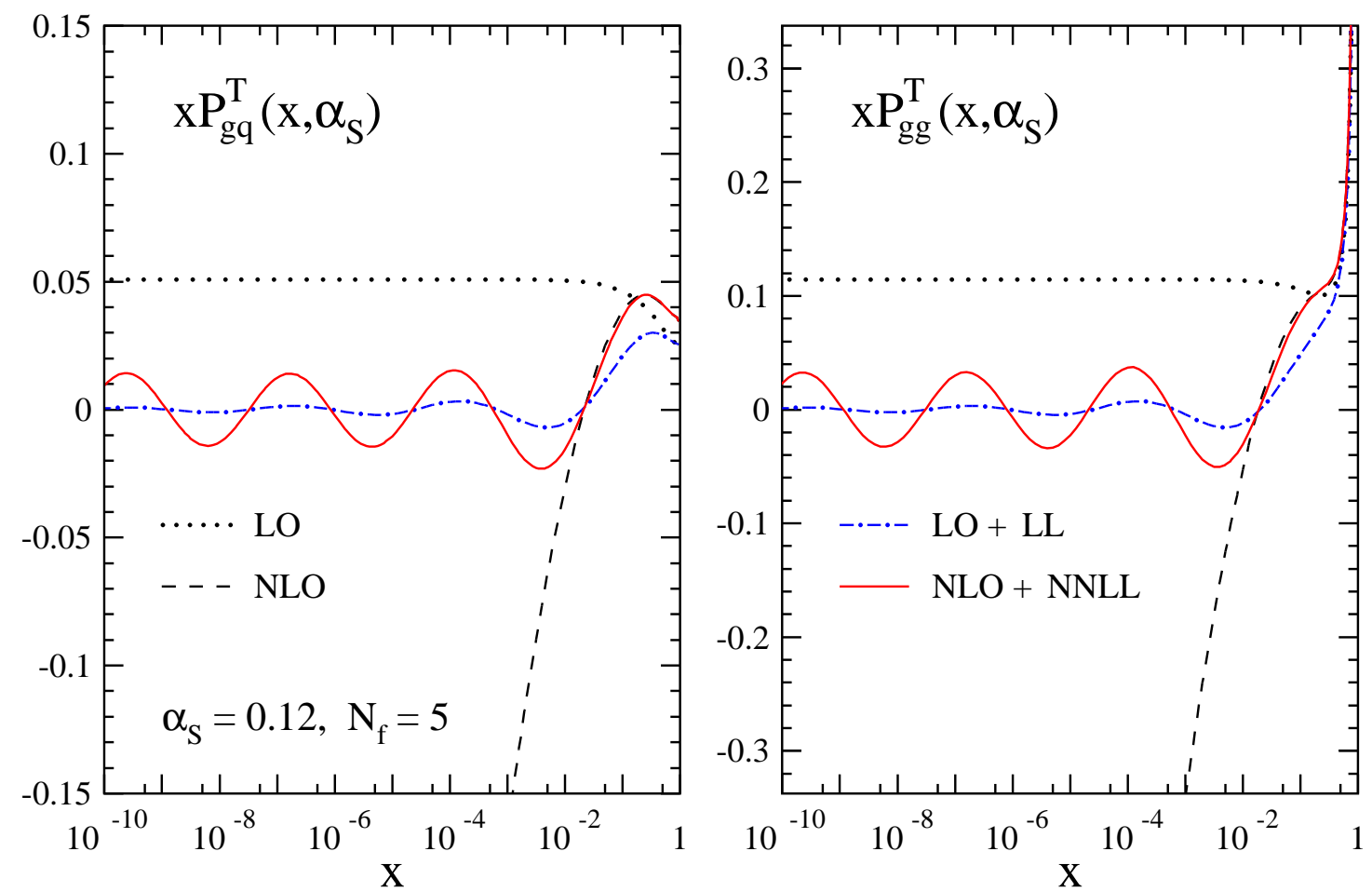

Figure 1: The timelike gluon-quark and gluon-gluon splitting functions (multiplied by $x$ ), shown for a very wide range of the momentum fraction $x$ at a typical value of the strong coupling constant $\alpha_{s}$. The all- $x$ (minimal $N=1$ finite) 'LO + resummed' and 'NLO + resummed' approximations are compared to the corresponding LO and NLO results valid only at large $x$, e.g., $x \gtrsim 10^{-2}$ for NLO.
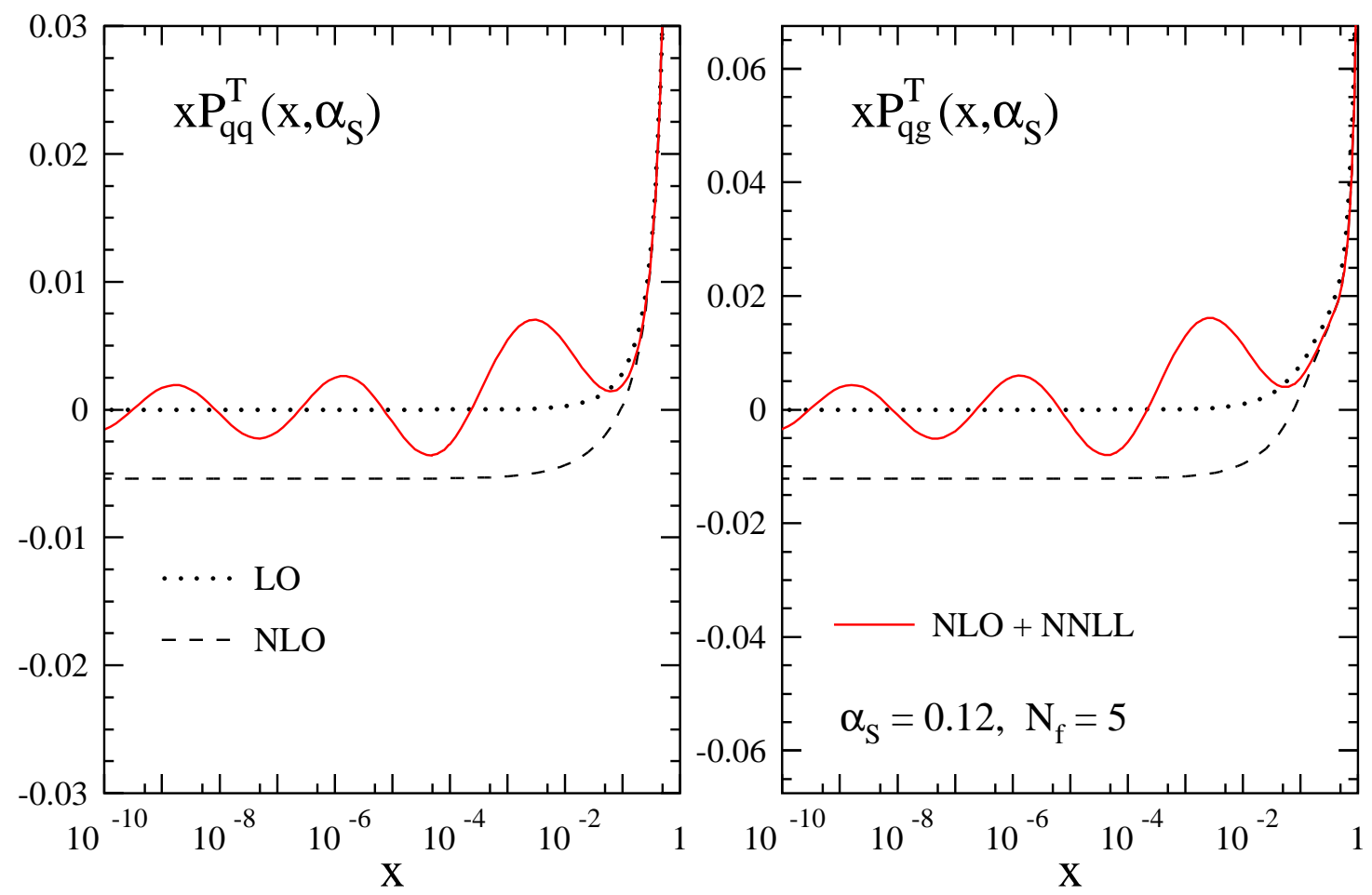

Figure 2: As Fig. 1, but for the timelike quark-quark and quark-gluon splitting functions, for which the LO contributions does not include $1 / x$ terms and the resummation starts at NLL level. 

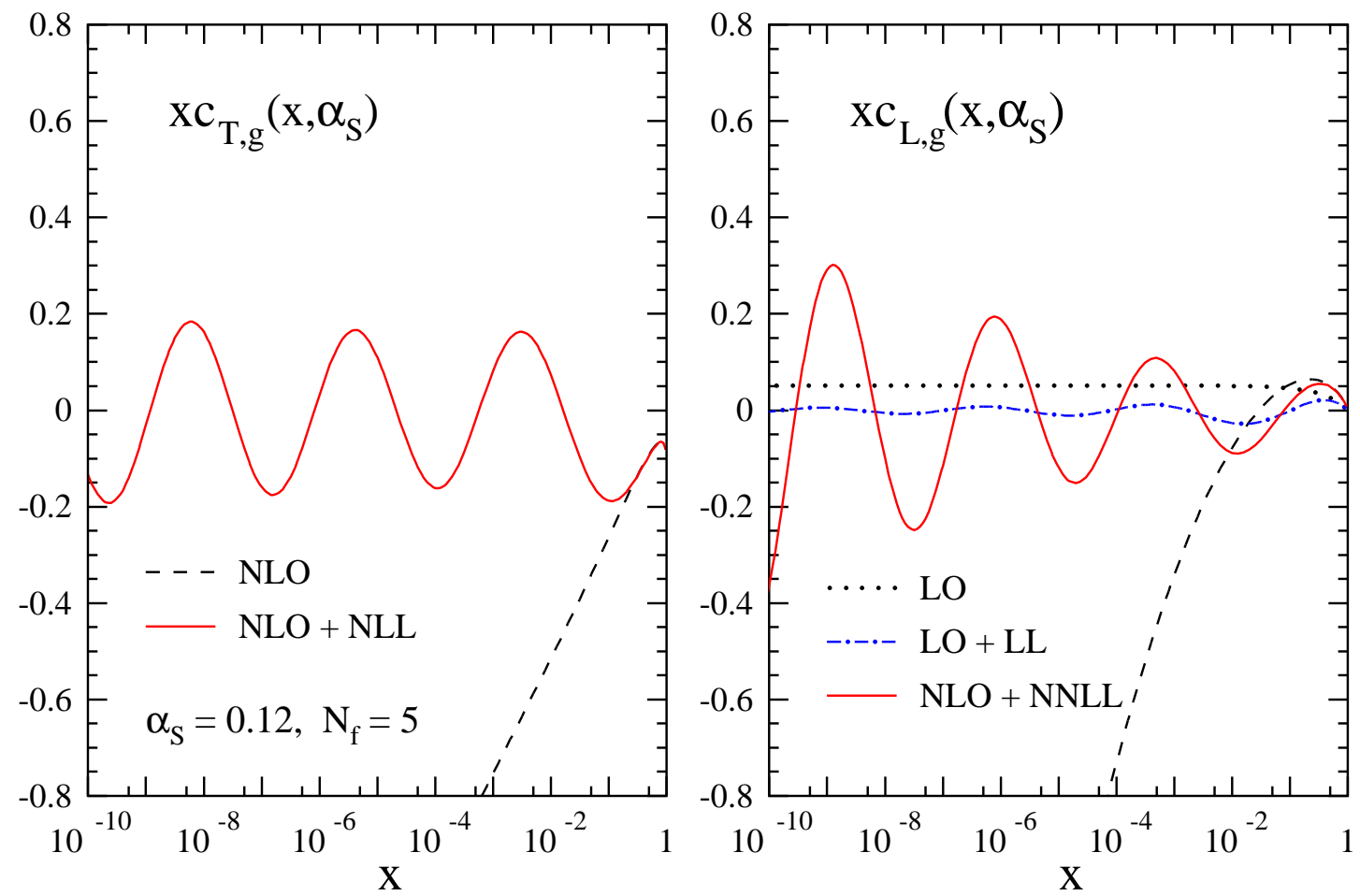

Figure 3: The all- $x$ gluon coefficient functions for the fragmentation functions $F_{T}$ and $F_{L}$ (multiplied by $x$ ), down to extremely small values of the scaling variable $x$. As discussed above Eqs. (4.1) and (5.1), the respective '(N)LO + resummed' approximations are defined by resumming as many logarithms as required to remove all $1 / x$ terms due to the $(\mathrm{N}) \mathrm{LO}$ contributions.
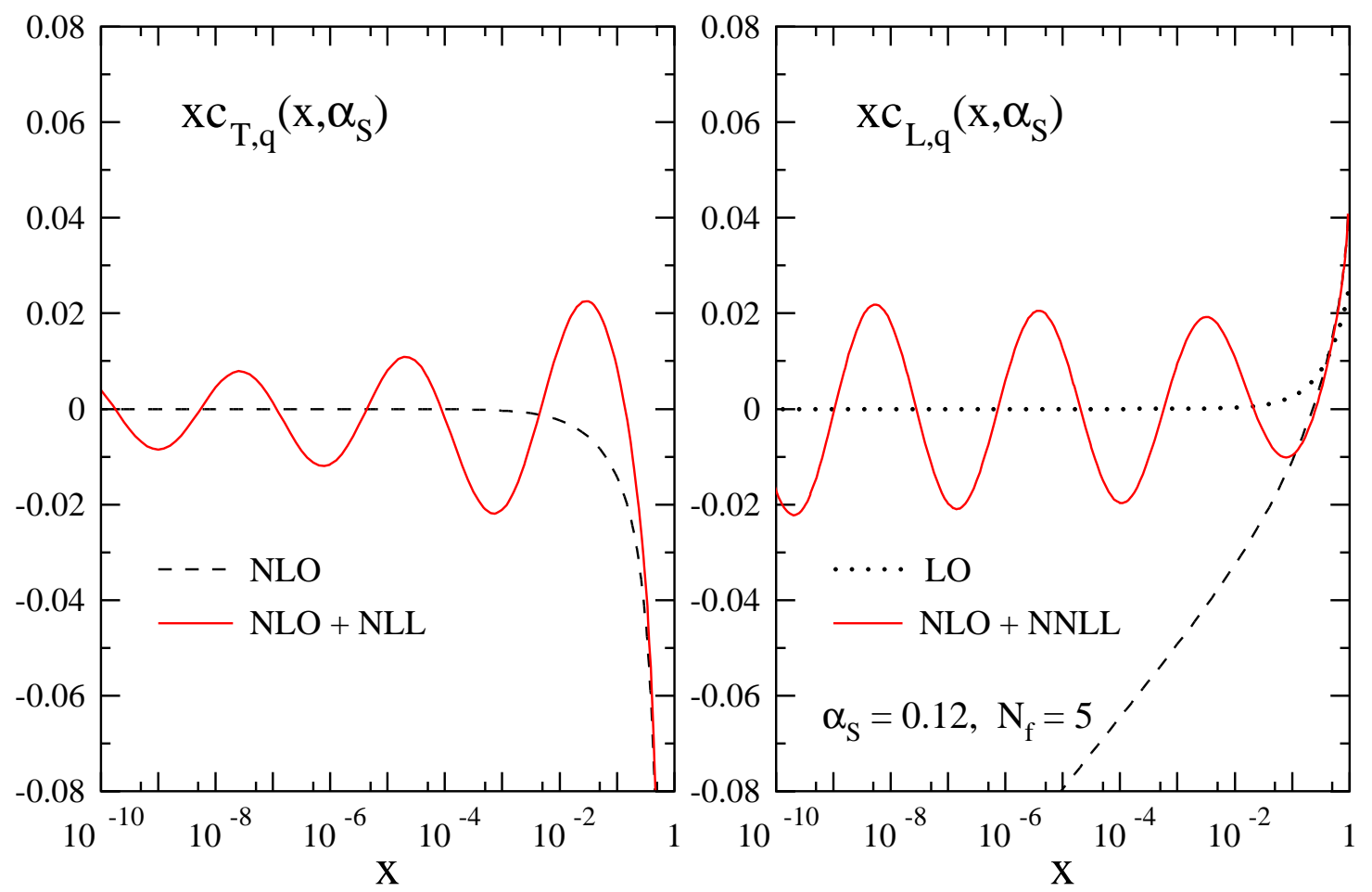

Figure 4: As Fig. 3, but for the quark coefficient functions which are suppressed by one power of $\ln x$ w.r.t. the gluon quantities. As the diagonal splitting functions, $C_{T, \mathrm{q}}$ includes a $\delta(1-x)$ term. 


\section{Summary}

We have presented the analytic all-order expressions in Mellin- $N$ space for the next-to-next-toleading logarithmic (NNLL) small- $x$ resummation of the splitting functions $P_{j i}^{T}$ for parton fragmentation and the coefficient functions for the fragmentation functions $F_{T}, F_{L}$ and $F_{\phi}$ in gauge-boson and (heavy-top) Higgs-exchange semi-inclusive $e^{+} e^{-}$annihilation. The resummation replaces the double-logarithmic $x^{-1} \ln ^{n} x$ small- $x$ enhancement of the fixed-order results for all these quantities by an oscillatory behaviour which can be described in terms of Bessel functions for the splitting

functions $P_{j i}^{T}\left(x, \alpha_{\mathrm{s}}\right)$. The present results are sufficient to construct a combined 'NLO + resummed' approximations which is applicable down to extremely small values of $x$ and determine the first three terms in the expansion of the first moments related to particle multiplicities in powers of $\sqrt{\alpha_{s}}$.

In view of the dependence of the oscillation amplitudes on the logarithmic order and the large size of the $N=1$ expansion parameter, an extension of the present results to the fifth $\left(\mathrm{N}^{4} L\right) \log$ arithms and a 'NNLO + resummed' approximation is desirable. Completing these results is well beyond our present approach based on the $D$-dimensional structure of the unfactorized fragmentation functions and the mass-factorization relations. However, we have taken a first step in this direction by confirming the all-order relation between initial-state and final-state splitting functions suggested in Refs. [21,22] at the NNLL level and applying it to predict the 'non-singlet' $C_{F}=0$ part of $P_{g g}^{T}$ at $\mathrm{N}^{4} \mathrm{LL}$ accuracy.

A FORM file of our NNLL results presented in the Sections 3, 4 and 5 above and Eqs. (B.7) (B.10) below can be obtained by downloading the source of this article from the arXiv servers.

\section{Acknowledgements}

A.V. and K.Y. are grateful to the organizers for the invitation to and hospitality at the workshop 'Loops and Legs in Quantum Field Theory', Wernigerode (Germany), April 2012. Without this meeting the vital contribution of K.Y. to this article, the derivation of Eqs. (A.1) and (A.2), would not have occurred. We have profited from the FORM efficiency improvements provided by Jos Vermaseren during the preparation of Ref. [19]. This research has been supported by the UK Science $\&$ Technology Facilities Council (STFC) under grant number ST/G00062X/1, the European-Union funded network LHCPhenoNet with contract number PITN-GA-2010-264564 and a Discovery Grant of the Natural Sciences and Engineering Research Council (NSERC) of Canada. 


\section{Appendix A}

The crucial step towards obtaining the analytic expressions presented above and in Appendix B was the solution of the sequence $A_{q i}^{(n)}$ in Ref. [19]. The key to understanding this sequence was the observation that the denominators began as, and subsequently were divisors of, the sequence of least common multiples of triangular numbers [36]. This suggested expanding each term as a sum of reciprocals of triangular numbers, which after some 'playing' with the initial terms led to

$$
\begin{aligned}
& A_{q i}^{(2)}=1=\frac{1}{1}, \\
& A_{q i}^{(3)}=\frac{11}{3}=\frac{2}{3}+\frac{3}{1}, \\
& A_{q i}^{(4)}=\frac{73}{6}=\frac{5}{6}+\frac{7}{3}+\frac{9}{1}, \\
& A_{q i}^{(5)}=\frac{1207}{30}=\frac{14}{10}+\frac{19}{6}+\frac{23}{3}+\frac{28}{1}, \\
& A_{q i}^{(6)}=\frac{2015}{15}=\frac{42}{15}+\frac{56}{10}+\frac{66}{6}+\frac{76}{3}+\frac{90}{1}, \ldots
\end{aligned}
$$

and the recognition that the successive numerators are the coefficients of the series [37] which are

some particular sums of products of Catalan numbers. Our knowledge of $A_{q i}^{(n)}$ to $n=17$ checks this identification to the $136^{\text {th }}$ entries in the sequences [36] and [37], virtually excluding an agreement by coincidence. Using MAPLE, this result was rewritten in the more accessible form

$$
A_{q i}^{(n)}=\frac{2(2 n-2) !}{(n-1) !(n+1) !}\left(\frac{1}{n-1}+\frac{1}{n}+\frac{6}{n+1}-2\right)+\frac{2(2 n) !}{n !(n+1) !} \sum_{k=n}^{2 n-3} \frac{1}{k},
$$

which was then understood to represent the expansion coefficients [in terms of $\xi$ in Eq. (3.1)] of the first line in Eq. (3.2). Once this function (the simplicity of which further supports the correctness of the above deductions) was known, it was not too difficult to derive all of Eqs. (3.2) - (3.5).

The corresponding $x$-space series can be readily obtained using Eqs. (A.2) and (7.1). We have not been able to express the result as a known special function. It may be interesting for future studies, however, that the function $\mathcal{L}$ in Eq. (3.1) has a simple Mellin (and Laplace) inverse, viz

$$
\int_{0}^{1} d x x^{N-2} \frac{1}{\ln x}\left(J_{0}(2 \sqrt{a} \ln x)-1\right)=\ln \left(\frac{1}{2}+\frac{1}{2} \sqrt{1+\frac{4 a}{(N-1)^{2}}}\right)
$$

with, in our case, $a=8 C_{A} a_{\mathrm{s}}$. In view of Eq. (7.2) and the logarithmic $x$-space arguments, the Mellin inverse of the first line of Eq. (3.2) and other products of the form $(N-1)^{n}\left(S^{z}-1\right) \mathcal{L}$ with $z=1,-1,-3, \ldots$, can hence be expressed as Laplace convolutions of Bessel functions.

\section{Appendix B}

Finally we present various, mostly lengthy results beyond the (at $N \neq 1)$ main 'NLO + resummed' approximation. We start with the $\mathrm{N}^{3} \mathrm{LL}$ corrections to the splitting functions $P_{\mathrm{qq}}^{T}$ and $P_{\mathrm{qg}}^{T}$ given at NNLL accuracy in Eqs. (3.2) and (3.3), 


$$
\begin{aligned}
& P_{\mathrm{qq}, \mathrm{N}^{3} \mathrm{LL}}^{T}(N)=\frac{1}{2592} \frac{C_{F} n_{f}}{C_{A}^{4}} a_{\mathrm{s}}^{2}\left\{-3\left(121 C_{A}^{4}+44 C_{A}^{3} n_{f}-88 C_{A}^{2} C_{F} n_{f}+4 C_{A}^{2} n_{f}^{2}-16 C_{A} C_{F} n_{f}^{2}\right.\right. \\
& \left.+16 C_{F}^{2} n_{f}^{2}\right) \frac{1}{\xi^{2}}\left(\left(S^{-2}-1-4 \xi-16 \xi^{2}\right)-\left(S^{-3}-1-6 \xi\right) \mathcal{L}\right)+6\left(341 C_{A}^{4}+40 C_{A}^{3} n_{f}+8 C_{A}^{2} C_{F} n_{f}\right. \\
& \left.-4 C_{A}^{2} n_{f}^{2}+32 C_{A} C_{F} n_{f}^{2}-48 C_{F}^{2} n_{f}^{2}\right) \frac{1}{\xi^{2}}\left(S^{-1}-1-2 \xi-6 \xi^{2}\right)+2\left(\left[905+4608 \zeta_{2}\right] C_{A}^{4}+3456 C_{A}^{3} C_{F}\right. \\
& \left.-1576 C_{A}^{3} n_{f}+8680 C_{A}^{2} C_{F} n_{f}+556 C_{A}^{2} n_{f}^{2}-2336 C_{A} C_{F} n_{f}^{2}+2064 C_{F}^{2} n_{f}^{2}\right) \frac{1}{\xi^{2}}\left(S-1+2 \xi+2 \xi^{2}\right) \\
& +3\left(\left[709-576 \zeta_{2}\right] C_{A}^{4}-300 C_{A}^{3} n_{f}+936 C_{A}^{2} C_{F} n_{f}-76 C_{A}^{2} n_{f}^{2}+400 C_{A} C_{F} n_{f}^{2}-496 C_{F}^{2} n_{f}^{2}\right) \frac{1}{\xi^{2}}\left(S^{-1}\right. \\
& -1-2 \xi) \mathcal{L}-24\left(55 C_{A}^{4}-12 C_{A}^{3} n_{f}+46 C_{A}^{2} C_{F} n_{f}-4 C_{A}^{2} n_{f}^{2}+20 C_{A} C_{F} n_{f}^{2}-24 C_{F}^{2} n_{f}^{2}\right) \frac{1}{\xi^{2}}\left(S^{-1}\right. \\
& -1-2 \xi) \mathcal{L}^{2}-6\left(\left[5231-6048 \zeta_{2}\right] C_{A}^{4}-\left[5184-6912 \zeta_{2}\right] C_{A}^{3} C_{F}-896 C_{A}^{3} n_{f}-2488 C_{A}^{2} C_{F} n_{f}\right. \\
& \left.-292 C_{A}^{2} n_{f}^{2}+2176 C_{A} C_{F} n_{f}^{2}-3440 C_{F}^{2} n_{f}^{2}\right) \frac{1}{\xi^{2}}(S-1+2 \xi) \mathcal{L}-24\left(\left[961-864 \zeta_{2}\right] C_{A}^{4}+\left[1152 \zeta_{2}\right.\right. \\
& \left.-612] C_{A}^{3} C_{F}-336 C_{A}^{3} n_{f}+386 C_{A}^{2} C_{F} n_{f}+4 C_{A}^{2} n_{f}^{2}+92 C_{A} C_{F} n_{f}^{2}-232 C_{F}^{2} n_{f}^{2}\right) \frac{1}{\xi^{2}}(S-1+2 \xi) \mathcal{L}^{2} \\
& -64\left(\left[6+18 \zeta_{2}\right] C_{A}^{4}+27 C_{A}^{3} C_{F}-29 C_{A}^{3} n_{f}+100 C_{A}^{2} C_{F} n_{f}+8 C_{A}^{2} n_{f}^{2}-44 C_{A} C_{F} n_{f}^{2}+60 C_{F}^{2} n_{f}^{2}\right) \\
& \cdot \frac{1}{\xi^{2}}(S-1) \mathcal{L}^{3}+192\left(\left[175-288 \zeta_{2}\right] C_{A}^{4}-\left[261-288 \zeta_{2}\right] C_{A}^{3} C_{F}+41 C_{A}^{3} n_{f}-318 C_{A}^{2} C_{F} n_{f}\right. \\
& \left.-28 C_{A}^{2} n_{f}^{2}+176 C_{A} C_{F} n_{f}^{2}-256 C_{F}^{2} n_{f}^{2}\right) \frac{1}{\xi} \mathcal{L}^{2}+8\left(\left[3659-9216 \zeta_{2}\right] C_{A}^{4}-\left[5832-8640 \zeta_{2}\right] C_{A}^{3} C_{F}\right. \\
& \left.-628 C_{A}^{3} n_{f}-3488 C_{A}^{2} C_{F} n_{f}-380 C_{A}^{2} n_{f}^{2}+2560 C_{A} C_{F} n_{f}^{2}-3696 C_{F}^{2} n_{f}^{2}\right) \frac{1}{\xi}(S-1+2 \xi) \\
& +12\left(\left[2017+576 \zeta_{2}\right] C_{A}^{4}+1728 C_{A}^{3} C_{F}-1772 C_{A}^{3} n_{f}+5432 C_{A}^{2} C_{F} n_{f}+356 C_{A}^{2} n_{f}^{2}\right. \\
& \left.-1744 C_{A} C_{F} n_{f}^{2}+2064 C_{F}^{2} n_{f}^{2}\right) \frac{1}{\xi}(S-1) \mathcal{L}-384\left(\left[117-252 \zeta_{2}\right] C_{A}^{4}-\left[207-288 \zeta_{2}\right] C_{A}^{3} C_{F}\right. \\
& \left.\left.+6 C_{A}^{3} n_{f}-164 C_{A}^{2} C_{F} n_{f}-14 C_{A}^{2} n_{f}^{2}+96 C_{A} C_{F} n_{f}^{2}-144 C_{F}^{2} n_{f}^{2}\right)\left(1+\frac{1}{\xi} \mathcal{L}\right)\right\}
\end{aligned}
$$

and

$$
\begin{aligned}
P_{\mathrm{qg}, \mathrm{N}}^{3} \mathrm{LL} & (N)=\frac{C_{A}}{C_{F}} P_{\mathrm{qq}, \mathrm{N}^{3} \mathrm{LL}}^{T}(N)+\frac{1}{54} \frac{n_{f}}{C_{A}^{3}} a_{\mathrm{s}}^{2}\left\{2 \left(\left[81-144 \zeta_{2}\right] C_{A}^{4}-90 C_{A}^{3} C_{F}+144 \zeta_{2} C_{A}^{3} C_{F}\right.\right. \\
& \left.-79 C_{A}^{3} n_{f}+106 C_{A}^{2} C_{F} n_{f}+6 C_{A}^{2} n_{f}^{2}-24 C_{A} C_{F} n_{f}^{2}+24 C_{F}^{2} n_{f}^{2}\right) \frac{1}{\xi^{2}}\left(S-1+2 \xi+2 \xi^{2}\right) \\
& -2\left(\left[483-576 \zeta_{2}\right] C_{A}^{4}-\left[360-576 \zeta_{2}\right] C_{A}^{3} C_{F}-139 C_{A}^{3} n_{f}+102 C_{A}^{2} C_{F} n_{f}+56 C_{A} C_{F} n_{f}^{2}\right. \\
& \left.-6 C_{A}^{2} n_{f}^{2}-88 C_{F}^{2} n_{f}^{2}\right) \frac{1}{\xi}(S-1+2 \xi)+\left(\left[429-576 \zeta_{2}\right] C_{A}^{4}-\left[360-576 \zeta_{2}\right] C_{A}^{3} C_{F}-213 C_{A}^{3} n_{f}\right. \\
& \left.+250 C_{A}^{2} C_{F} n_{f}-2 C_{A}^{2} n_{f}^{2}+40 C_{A} C_{F} n_{f}^{2}-72 C_{F}^{2} n_{f}^{2}\right) \frac{1}{\xi^{2}}(S-1+2 \xi) \mathcal{L}+8\left(\left[137-144 \zeta_{2}\right] C_{A}^{4}\right. \\
& \left.-\left[90-144 \zeta_{2}\right] C_{A}^{3} C_{F}-17 C_{A}^{3} n_{f}-6 C_{A}^{2} C_{F} n_{f}-6 C_{A}^{2} n_{f}^{2}+36 C_{A} C_{F} n_{f}^{2}-48 C_{F}^{2} n_{f}^{2}\right)\left(1+\frac{1}{\xi} \mathcal{L}\right) \\
& +\left(11 C_{A}^{4}+13 C_{A}^{3} n_{f}-26 C_{A}^{2} C_{F} n_{f}+2 C_{A}^{2} n_{f}^{2}-8 C_{A} C_{F} n_{f}^{2}+8 C_{F}^{2} n_{f}^{2}\right) \frac{1}{\xi^{2}}\left(S^{-1}-1-2 \xi\right) \mathcal{L} \\
& +4\left(\left[59-72 \zeta_{2}\right] C_{A}^{4}-\left[45-72 \zeta_{2}\right] C_{A}^{3} C_{F}-20 C_{A}^{3} n_{f}+20 C_{A}^{2} C_{F} n_{f}-2 C_{A}^{2} n_{f}^{2}+14 C_{A} C_{F} n_{f}^{2}\right. \\
& \left.\left.-20 C_{F}^{2} n_{f}^{2}\right) \frac{1}{\xi^{2}}(S-1) \mathcal{L}^{2}\right\} .
\end{aligned}
$$

Together with the first moments of the corresponding truncated second-order splitting functions

$$
\begin{aligned}
& \bar{P}_{\mathrm{qq}}^{T(1)}(N=1)=-\left(13-12 \zeta_{2}+8 \zeta_{3}\right) C_{A} C_{F}+\left(26-24 \zeta_{2}+16 \zeta_{3}\right) C_{F}^{2}+\frac{634}{27} C_{F} n_{f}, \\
& \bar{P}_{\mathrm{qg}}^{T(1)}(N=1)=\left(\frac{796}{27}-\frac{16}{3} \zeta_{2}\right) C_{A} n_{f}+4 C_{F} n_{f},
\end{aligned}
$$


which can be derived, e.g., using FORM packages [24] based on Refs. [38, 39] as discussed in Ref. [19], Eqs. (B.1) and (B.2) lead to the combined results

$$
\begin{aligned}
\left.P_{\mathrm{qq}}^{T}(N=1)\right|_{a_{\mathrm{s}}^{2}}= & \frac{1}{162} \frac{C_{F}}{C_{A}^{4}}\left\{-\left[2106-1944 \zeta_{2}+1296 \zeta_{3}\right] C_{A}^{5}+\left[4477-2016 \zeta_{2}\right] C_{A}^{4} n_{f}\right. \\
& +\left[4212-3888 \zeta_{2}+2592 \zeta_{3}\right] C_{A}^{4} C_{F}-1124 C_{A}^{3} n_{f}^{2}+1728 \zeta_{2} C_{A}^{3} C_{F} n_{f} \\
& \left.+116 C_{A}^{2} n_{f}^{3}+2336 C_{A}^{2} C_{F} n_{f}^{2}-448 C_{A} C_{F} n_{f}^{3}+432 C_{F}^{2} n_{f}^{3}\right\}, \\
\left.P_{\mathrm{qg}}^{T}(N=1)\right|_{a_{\mathrm{s}}^{2}}= & \frac{1}{162} \frac{n_{f}}{C_{A}^{3}}\left\{\left[3913-1152 \zeta_{2}\right] C_{A}^{4}+1728 C_{A}^{3} C_{F}-812 C_{A}^{3} n_{f}+116 C_{A}^{2} n_{f}^{2}\right. \\
& \left.+2240 C_{A}^{2} C_{F} n_{f}-544 C_{A} C_{F} n_{f}^{2}+624 C_{F}^{2} n_{f}^{2}\right\} .
\end{aligned}
$$

The numerical values for $n_{f}=5$ of these coefficients have been included in Eq. (3.8) above.

The NNLL corrections to the coefficient functions (4.1) - (4.4) for $F_{T}$ and $F_{\phi}$ can be written as

$$
\begin{aligned}
& C_{T, \mathrm{q}, \mathrm{NNL}}(N)=\frac{1}{432} \frac{C_{F} n_{f}}{C_{A}^{3}} a_{\mathrm{s}}\left\{-192\left(5 C_{A}^{2}-4 C_{A} n_{f}+12 C_{F} n_{f}\right)\left(\frac{1}{\xi} \mathcal{L}+1\right)+8\left(35 C_{A}^{2}-14 C_{A} n_{f}\right.\right. \\
& \left.+24 C_{F} n_{f}\right) \frac{1}{\xi}\left(F^{-3}-1+3 \xi\right)-8\left(35 C_{A}^{2}+34 C_{A} n_{f}-120 C_{F} n_{f}\right) \frac{1}{\xi}\left(F^{-1}-1+\xi\right)-3\left(505 C_{A}^{2}\right. \\
& \left.-150 C_{A} n_{f}+416 C_{F} n_{f}\right) \frac{1}{\xi}\left(F^{-1}-1\right) \mathcal{L}-4\left(887 C_{A}^{2}-122 C_{A} n_{f}+336 C_{F} n_{f}\right)(F-1) \\
& +4\left(169 C_{A}^{2}+86 C_{A} n_{f}-276 C_{F} n_{f}\right) \frac{1}{\xi}(F-1) \mathcal{L}+12\left(11 C_{A}^{2}+2 C_{A} n_{f}-4 C_{F} n_{f}\right)\left(4\left(F^{3}-1\right)\right. \\
& \left.-\frac{1}{\xi}\left(F^{5}-1\right) \mathcal{L}\right)+\left(11 C_{A}^{2}-2 C_{A} n_{f}\right)\left(20\left(F^{5}-1\right)+6 \frac{1}{\xi}\left(F^{3}-1\right) \mathcal{L}-5 \frac{1}{\xi}\left(F^{7}-1\right) \mathcal{L}\right) \\
& \left.+96\left(5 C_{A}^{2}-2 C_{A} n_{f}+6 C_{F} n_{f}\right) \frac{1}{\xi} F \mathcal{L}^{2}\right\} \\
& C_{T, \mathrm{~g}, \mathrm{NNL}}(N)=-\frac{C_{F}}{C_{A}} C_{T \mathrm{q}, \mathrm{NNL}}(N)+\frac{1}{82944} \frac{C_{F}}{C_{A}^{4}} a_{\mathrm{s}}\left\{9 2 1 6 \left(\left[54-72 \zeta_{2}\right] C_{A}^{4}-\left[45-72 \zeta_{2}\right] C_{A}^{3} C_{F}\right.\right. \\
& \left.-23 C_{A}^{3} n_{f}+20 C_{A}^{2} C_{F} n_{f}\right)\left(1-\frac{1}{\xi}(S-1+2 \xi-\mathcal{L})\right)-36864\left(C_{A}^{2} C_{F} n_{f}+C_{A} C_{F} n_{f}^{2}\right. \\
& \left.-2 C_{F}^{2} n_{f}^{2}\right) \frac{1}{\xi}((S-1+2 \xi)+(F-1) \mathcal{L})+256\left(\left[637-1620 \zeta_{2}\right] C_{A}^{4}-\left[810-1296 \zeta_{2}\right] C_{A}^{3} C_{F}\right. \\
& \left.-206 C_{A}^{3} n_{f}+129 C_{A}^{2} C_{F} n_{f}-44 C_{A}^{2} n_{f}^{2}+258 C_{A} C_{F} n_{f}^{2}-342 C_{F}^{2} n_{f}^{2}\right) \frac{1}{\xi}\left(F^{-3}-1+3 \xi\right) \\
& +256\left(\left[1523-972 \zeta_{2}\right] C_{A}^{4}-\left[810-1296 \zeta_{2}\right] C_{A}^{3} C_{F}-262 C_{A}^{3} n_{f}+159 C_{A}^{2} C_{F} n_{f}-28 C_{A}^{2} n_{f}^{2}\right. \\
& \left.+318 C_{A} C_{F} n_{f}^{2}-522 C_{F}^{2} n_{f}^{2}\right) \frac{1}{\xi}\left(F^{-1}-1+\xi\right)+\left(\left[1285825-1327104 \zeta_{2}\right] C_{A}^{4}-331776\right. \\
& \cdot\left[1-2 \zeta_{2}\right] C_{A}^{3} C_{F}-24716 C_{A}^{3} n_{f}-19392 C_{A}^{2} C_{F} n_{f}-45020 C_{A}^{2} n_{f}^{2}+233088 C_{A} C_{F} n_{f}^{2} \\
& \left.-290304 C_{F}^{2} n_{f}^{2}\right)(F-1)+8\left(14839 C_{A}^{4}+1168 C_{A}^{3} n_{f}-2172 C_{A}^{2} C_{F} n_{f}-284 C_{A}^{2} n_{f}^{2}\right. \\
& \left.+1128 C_{A} C_{F} n_{f}^{2}-1152 C_{F}^{2} n_{f}^{2}\right)\left(F^{3}-1\right)+\left(\left[75461+165888 \zeta_{2}\right] C_{A}^{4}+41156 C_{A}^{3} n_{f}\right. \\
& \left.-110016 C_{A}^{2} C_{F} n_{f}+11444 C_{A}^{2} n_{f}^{2}-54144 C_{A} C_{F} n_{f}^{2}+62208 C_{F}^{2} n_{f}^{2}\right)\left(F^{5}-1\right)+16\left(11957 C_{A}^{4}\right. \\
& \left.-40 C_{A}^{3} n_{f}-4488 C_{A}^{2} C_{F} n_{f}-388 C_{A}^{2} n_{f}^{2}+816 C_{A} C_{F} n_{f}^{2}\right)\left(F^{7}-1\right)-\left(21901 C_{A}^{4}+55396 C_{A}^{3} n_{f}\right. \\
& \left.-63360 C_{A}^{2} C_{F} n_{f}+724 C_{A}^{2} n_{f}^{2}-11520 C_{A} C_{F} n_{f}^{2}+11520 C_{F}^{2} n_{f}^{2}\right)\left(F^{9}-1\right)-840\left(121 C_{A}^{4}\right. \\
& \left.\left.-44 C_{A}^{2} C_{F} n_{f}-4 C_{A}^{2} n_{f}^{2}+8 C_{A} C_{F} n_{f}^{2}\right)\left(F^{11}-1\right)-385 C_{A}^{2}\left(11 C_{A}-2 n_{f}\right)^{2}\left(F^{13}-1\right)\right\}
\end{aligned}
$$


and

$$
\begin{aligned}
C_{\phi, \mathrm{g}, \mathrm{NNL}}(N)=-C_{T \mathrm{q}, \mathrm{NNL}}(N)+\frac{1}{82944} \frac{1}{C_{A}^{3}} a_{\mathrm{s}}\left\{2 5 6 \left(\left[601-324 \zeta_{2}\right] C_{A}^{4}+64 C_{A}^{3} n_{f}+93 C_{A}^{2} C_{F} n_{f}\right.\right. \\
\left.\quad-8 C_{A}^{2} n_{f}^{2}-30 C_{A} C_{F} n_{f}^{2}+90 C_{F}^{2} n_{f}^{2}\right) \frac{1}{\xi}\left((S-1) F^{-1}+2 \xi\right)-18432\left(2 C_{A}^{2} C_{F} n_{f}-C_{A} C_{F} n_{f}^{2}\right. \\
\left.\quad+2 C_{F}^{2} n_{f}^{2}\right) \frac{1}{\xi}(S-1) F \mathcal{L}+\left(\left[3335233-663552 \zeta_{2}\right] C_{A}^{4}-162380 C_{A}^{3} n_{f}-356928 C_{A}^{2} C_{F} n_{f}\right. \\
\left.\quad-7004 C_{A}^{2} n_{f}^{2}+46464 C_{A} C_{F} n_{f}^{2}-69120 C_{F}^{2} n_{f}^{2}\right)(F-1)+\left(9559 C_{A}^{4}+3256 C_{A}^{3} n_{f}+244 C_{A}^{2} n_{f}^{2}\right. \\
\left.\quad-6732 C_{A}^{2} C_{F} n_{f}-1080 C_{A} C_{F} n_{f}^{2}+1152 C_{F}^{2} n_{f}^{2}\right) 8\left(F^{3}-1\right)-\left(\left[55483-165888 \zeta_{2}\right] C_{A}^{4}\right. \\
\left.\quad-17540 C_{A}^{3} n_{f}+52032 C_{A}^{2} C_{F} n_{f}-4148 C_{A}^{2} n_{f}^{2}+30336 C_{A} C_{F} n_{f}^{2}-43776 C_{F}^{2} n_{f}^{2}\right)\left(F^{5}-1\right) \\
\quad+16\left(9317 C_{A}^{4}-\left(220 C_{A}^{3}+3168 C_{A}^{2} C_{F}\right) n_{f}-\left(268 C_{A}^{2}-576 C_{A} C_{F}\right) n_{f}^{2}\right)\left(F^{7}-1\right)-\left(21901 C_{A}^{4}\right. \\
\left.\quad+55396 C_{A}^{3} n_{f}-63360 C_{A}^{2} C_{F} n_{f}+724 C_{A}^{2} n_{f}^{2}-11520 C_{F} n_{f}^{2}\left(C_{A}-C_{F}\right)\right)\left(F^{9}-1\right)-\left(121 C_{A}^{4}\right. \\
\left.\left.\quad-44 C_{A}^{2} C_{F} n_{f}-4 C_{A}^{2} n_{f}^{2}+8 C_{A} C_{F} n_{f}^{2}\right) 840\left(F^{11}-1\right)-385\left(11 C_{A}-2 n_{f}\right)^{2} C_{A}^{2}\left(F^{13}-1\right)\right\}(\mathrm{B} .9) \\
\quad \\
C_{\phi, \mathrm{q}, \mathrm{NNL}}(N)=\frac{C_{A}}{C_{F}} C_{T \mathrm{q}, \mathrm{NNL}}(N)+\frac{2}{9} \frac{n_{f}}{C_{A}^{2}} a_{\mathrm{s}}\left\{-2\left(C_{A}^{2}+C_{A} n_{f}-2 C_{F} n_{f}\right)\left(1+\frac{1}{\xi} \mathcal{L}\right)\right. \\
\quad-\left(2 C_{A}^{2}-C_{A} n_{f}+2 C_{F} n_{f}\right) \frac{1}{\xi}\left(\left(F^{-3}-1+3 \xi\right)-\left(F^{-1}-1\right) \mathcal{L}\right) \\
\left.+\left(4 C_{A}^{2}+C_{A} n_{f}-2 C_{F} n_{f}\right) \frac{1}{\xi}\left(\left(F^{-1}-1+\xi\right)-(F-1) \mathcal{L}\right)\right\}
\end{aligned}
$$

Finally we write down the $\mathrm{N}^{4} \mathrm{LL}$ contribution to the splitting function $P_{\mathrm{gg}}^{T}$ in the limit $C_{F}=0$, which still contains one unknown coefficient as discussed above,

$$
\begin{aligned}
& \left.P_{\mathrm{gg}}^{T}(N)\right|_{\mathrm{N}^{4} \mathrm{LL}} ^{C_{F}=0}=\frac{1}{13271040} \frac{1}{C_{A}^{2}} a_{\mathrm{s}}^{2} \bar{N}\left\{1 6 \left(\left[15688235-19918080 \zeta_{2}+7983360 \zeta_{3}\right.\right.\right. \\
& \left.+5059584 \zeta_{2}^{2}\right] C_{A}^{4}+\left[914360+875520 \zeta_{2}-2142720 \zeta_{3}\right] C_{A}^{3} n_{f}-\left[134200+46080 \zeta_{2}\right] C_{A}^{2} n_{f}^{2} \\
& \left.+5600 n_{f}^{3} C_{A}-80 n_{f}^{4}\right)(S-1)-32\left(\left[7822505-2826000 \zeta_{2}+1330560 \zeta_{3}-134784 \zeta_{2}^{2}\right] C_{A}^{4}\right. \\
& \left.+\left[514490+83520 \zeta_{2}-276480 \zeta_{3}\right] C_{A}^{3} n_{f}+\left[16880+20160 \zeta_{2}\right] C_{A}^{2} n_{f}^{2}-2840 n_{f}^{3} C_{A}+80 n_{f}^{4}\right) \\
& \cdot \frac{1}{\xi}(S-1+2 \xi)+2\left(\left[12686895+12997440 \zeta_{2}-2471040 \zeta_{3}-10907136 \zeta_{2}^{2}\right] C_{A}^{4}\right. \\
& -\left[3309880+564480 \zeta_{2}-1624320 \zeta_{3}\right] C_{A}^{3} n_{f}+\left[37960-172800 \zeta_{2}\right] C_{A}^{2} n_{f}^{2}+21280 n_{f}^{3} C_{A} \\
& \left.-1040 n_{f}^{4}\right) \frac{1}{\xi^{2}}\left(S-1+2 \xi+2 \xi^{2}\right)-4\left(\left[3135445+6822720 \zeta_{2}+190080 \zeta_{3}-5868288 \zeta_{2}^{2}\right] C_{A}^{4}\right. \\
& -\left[1973120+587520 \zeta_{2}-1071360 \zeta_{3}\right] C_{A}^{3} n_{f}+\left[106520-149760 \zeta_{2}\right] C_{A}^{2} n_{f}^{2}+14080 n_{f}^{3} C_{A} \\
& \left.-1200 n_{f}^{4}\right) \frac{1}{\xi^{2}}\left(S^{-1}-1-2 \xi-6 \xi^{2}\right)-\left(\left[2095591+158976 \zeta_{2}-1140480 \zeta_{3}+331776 \zeta_{2}^{2}\right] C_{A}^{4}\right. \\
& \left.+\left[61560+396288 \zeta_{2}-207360 \zeta_{3}\right] C_{A}^{3} n_{f}-\left[83352-64512 \zeta_{2}\right] C_{A}^{2} n_{f}^{2}+224 n_{f}^{3} C_{A}+880 n_{f}^{4}\right) \\
& \cdot 5 \frac{1}{\xi^{2}}\left(S^{-3}-1-6 \xi-30 \xi^{2}\right)-10\left(\left[198803-209088 \zeta_{2}\right] C_{A}^{4}+\left[69872-76032 \zeta_{2}\right] C_{A}^{3} n_{f}\right. \\
& \left.-\left[600+6912 \zeta_{2}\right] C_{A}^{2} n_{f}^{2}-2368 n_{f}^{3} C_{A}-208 n_{f}^{4}\right) \frac{1}{\xi^{2}}\left(S^{-5}-1-10 \xi-70 \xi^{2}\right) \\
& \left.-25\left(11 C_{A}+2 n_{f}\right)^{4} \frac{1}{\xi^{2}}\left(S^{-7}-1-14 \xi-126 \xi^{2}\right)\right\}+C_{A}^{2} a_{\mathrm{s}}^{2} \bar{N}\left(S+S^{-1}-2\right) B_{\mathrm{gg}}^{S(3)},
\end{aligned}
$$


where the normalization of the last coefficient has been given below Eq. (6.8). This result leads to the first moment

$$
\begin{aligned}
\left.P_{\mathrm{gg}}^{T}(N=1)\right|_{a_{\mathrm{s}}^{5 / 2}} ^{C_{F}=0} & =\left(2 C_{A}\right)^{1 / 2} \frac{1}{41472} \frac{1}{C_{A}^{2}}\left\{\left[182872+175104 \zeta_{2}-428544 \zeta_{3}\right] C_{A}^{3} n_{f}\right. \\
& +\left[3137647-3983616 \zeta_{2}+1596672 \zeta_{3}+5059584 / 5 \zeta_{2}^{2}\right] C_{A}^{4} \\
& \left.-\left[26840+9216 \zeta_{2}\right] C_{A}^{2} n_{f}^{2}+1120 C_{A} n_{f}^{3}-16 n_{f}^{4}+165888 C_{A}^{4} B_{\mathrm{gg}}^{S(3)}\right\}
\end{aligned}
$$

which has been included in an approximate numerical form for $n_{f}=5 \mathrm{Eq}$. (6.8).

\section{References}

[1] P. Nason and B.R. Webber, Nucl. Phys. B421 (1994) 473

[2] J. Beringer et al. [Particle Data Group], Phys. Rev. D86 (2012) 010001

[3] J.R. Ellis, M.K. Gaillard and D.V. Nanopoulos, Nucl. Phys. B106 (1976) 292;

M.A. Shifman, A.I. Vainshtein, M.B. Voloshin and V.I. Zakharov, Sov. J. Nucl. Phys. 30 (1979) 711

[4] W. Furmanski and R. Petronzio, Phys. Lett. 97B (1980) 437

[5] P.J. Rijken and W.L. van Neerven, Phys. Lett. B386 (1996) 422, hep-ph/9604436;

Nucl. Phys. B488 (1997) 233, hep-ph/9609377

[6] A. Mitov, S. Moch and A. Vogt, Phys. Lett. B638 (2006) 61, hep-ph/0604053

[7] A. Mitov and S. Moch, Nucl. Phys. B751 (2006) 18, hep-ph/0604160

[8] S. Moch and A. Vogt, Phys. Lett. B659 (2008) 290, arXiv:0709.3899

[9] A.A. Almasy, S. Moch and A. Vogt, Nucl. Phys. B854 (2012) 133, arXiv:1107.2263

[10] E.B. Zijlstra and W.L. van Neerven, Phys. Lett. B272 (1991) 127; Phys. Lett. B273 (1991) 476

[11] S. Moch and J.A.M. Vermaseren, Nucl. Phys. B573 (2000) 853, hep-ph/9912355

[12] S. Moch, J.A.M. Vermaseren and A. Vogt, Nucl. Phys. B688 (2004) 101, hep-ph/0403192;

Nucl. Phys. B691 (2004) 129, hep-ph/0404111

[13] A.H. Mueller, Phys. Lett. B104 (1981) 161;

A. Bassetto, M. Ciafaloni, G. Marchesini and A.H. Mueller, Nucl. Phys. B207 (1982) 189

[14] A.H. Mueller, Nucl. Phys. B213 (1983) 85; (erratum in) Nucl. Phys. B241 (1984) 141

[15] J.B. Gaffney and A.H. Mueller, Nucl. Phys. B250 (1985) 109

[16] S. Albino, B.A. Kniehl, G. Kramer and W. Ochs, Phys. Rev. Lett. 95 (2005) 232002, hep-ph/0503170; Phys. Rev. D73 (2006) 054020, hep-ph/0510319

[17] S. Albino, P. Bolzoni, B.A. Kniehl and A. Kotikov, Nucl. Phys. B851 (2011) 86, arXiv:1104.3018; Nucl. Phys. B855 (2012) 801, arXiv:1108.3948

[18] P. Bolzoni, proceedings of DIS 2012 (Bonn, Germany, April 2012), arXiv:1206.3039

[19] A. Vogt, JHEP 10 (2011) 025, arXiv:1108.2993 
[20] R. Aloisio, V. Berezinsky and M. Kachelrieß, Phys. Rev. D69 (2004) 094023, hep-ph/0307279

[21] Yu.L. Dokshitzer, G. Marchesini and G.P. Salam, Phys. Lett. B634 (2006) 504, hep-ph/0511302

[22] G. Marchesini, Workshop on Future Prospects in QCD at High Energies (Brookhaven, USA, 2006), hep-ph/0605262;

[23] Yu.L. Dokshitzer and G. Marchesini, Phys. Lett. B646 (2007) 189, hep-ph/0612248

[24] J.A.M. Vermaseren, New features of FORM, math-ph/0010025;

M. Tentyukov and J. Vermaseren, Comput. Phys. Commun. 181 (2010) 1419, arXiv:hep-ph/0702279

[25] A.A. Almasy, G. Soar and A. Vogt, JHEP 03 (2011) 030, arXiv:1012.3352,

A.A. Almasy, N.A. Lo Presti and A. Vogt, PoS RADCOR 2011 (2012) 023, arXiv:1202.5224; to appear

[26] W. Furmanski and R. Petronzio, Z. Phys. C11 (1982) 293

[27] J. Blümlein, V. Ravindran, W.L. van Neerven, Nucl. Phys. B586 (2000) 349, hep-ph/0004172

[28] J.A.M. Vermaseren, A. Vogt and S. Moch, Nucl. Phys. B724 (2005) 3, hep-ph/0504242

[29] E.A. Kuraev, L.N. Lipatov and V.S. Fadin, Sov. Phys. JETP 45 (1977) 199;

I.I. Balitsky and L.N. Lipatov, Sov. J. Nucl. Phys. 28 (1978), 822

[30] T. Jaroszewicz, Phys. Lett. B116 (1982) 291;

S. Catani, F. Fiorani and G. Marchesini, Nucl. Phys. B336 (1990) 18

[31] V.S. Fadin and L.N. Lipatov, Phys. Lett. B429 (1998) 127, hep-ph/9802290;

M. Ciafaloni and G. Camici, Phys. Lett. B430 (1998) 349, hep-ph/9803389

[32] M. Ciafaloni and D. Colferai, JHEP 09 (2005) 069, hep-ph/0507106;

M. Ciafaloni, D. Colferai, G.P. Salam and A.M. Stasto, Phys. Lett. B635 (2006) 320, hep-ph/0601200

[33] R. D. Ball and S. Forte, Nucl. Phys. B742 (2006) 158, hep-ph/0601049;

S. Marzani, R. D. Ball, P. Falgari and S. Forte, Nucl. Phys. B783 (2007) 143, arXiv:0704.2404

[34] A. Vogt, Comput. Phys. Commun. 170 (2005) 65, hep-ph/0408244

[35] M. Abramowitz, I.A. Stegun (eds.), Handbook of Mathematical Functions, Dover (New York) 1965

[36] The On-Line Encyclopedia of Integer Sequences, published electronically at http://oeis .org, 2010, sequence A025555

[37] The On-Line Encyclopedia of Integer Sequences, sequence A028378; see also sequence A028364

[38] J.A.M. Vermaseren, Int. J. Mod. Phys. A14 (1999) 2037, hep-ph/9806280

[39] E. Remiddi and J.A.M. Vermaseren, Int. J. Mod. Phys. A15 (2000) 725, hep-ph/9905237 